\title{
Enhanced anti-inflammatory potential of degradation resistant curcumin/ferulic acid eutectics embedded in triglyceride-based microemulsions
}

Article

Accepted Version

Sanduk, F., Meng, Y., Widera, D. ORCID:

https://orcid.org/0000-0003-1686-130X, Kowalczyk, R. M., Michael, N., Kaur, A., Yip, V., Zulu, S., Zavrou, I., Hana, L., Yaqoob, M. and Al-Obaidi, H. (2020) Enhanced antiinflammatory potential of degradation resistant

curcumin/ferulic acid eutectics embedded in triglyceride-based microemulsions. Journal of Drug Delivery Science and Technology, 60. 102067. ISSN 1773-2247 doi: https://doi.org/10.1016/j.jddst.2020.102067 Available at https://centaur.reading.ac.uk/92984/

It is advisable to refer to the publisher's version if you intend to cite from the work. See Guidance on citing.

To link to this article DOI: http://dx.doi.org/10.1016/j.jddst.2020.102067

Publisher: Elsevier 
copyright holders. Terms and conditions for use of this material are defined in the End User Agreement.

\section{www.reading.ac.uk/centaur}

\section{CentAUR}

Central Archive at the University of Reading

Reading's research outputs online 


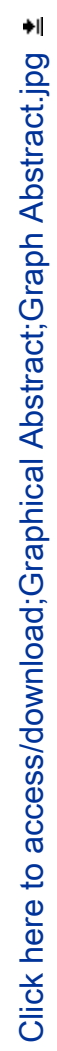

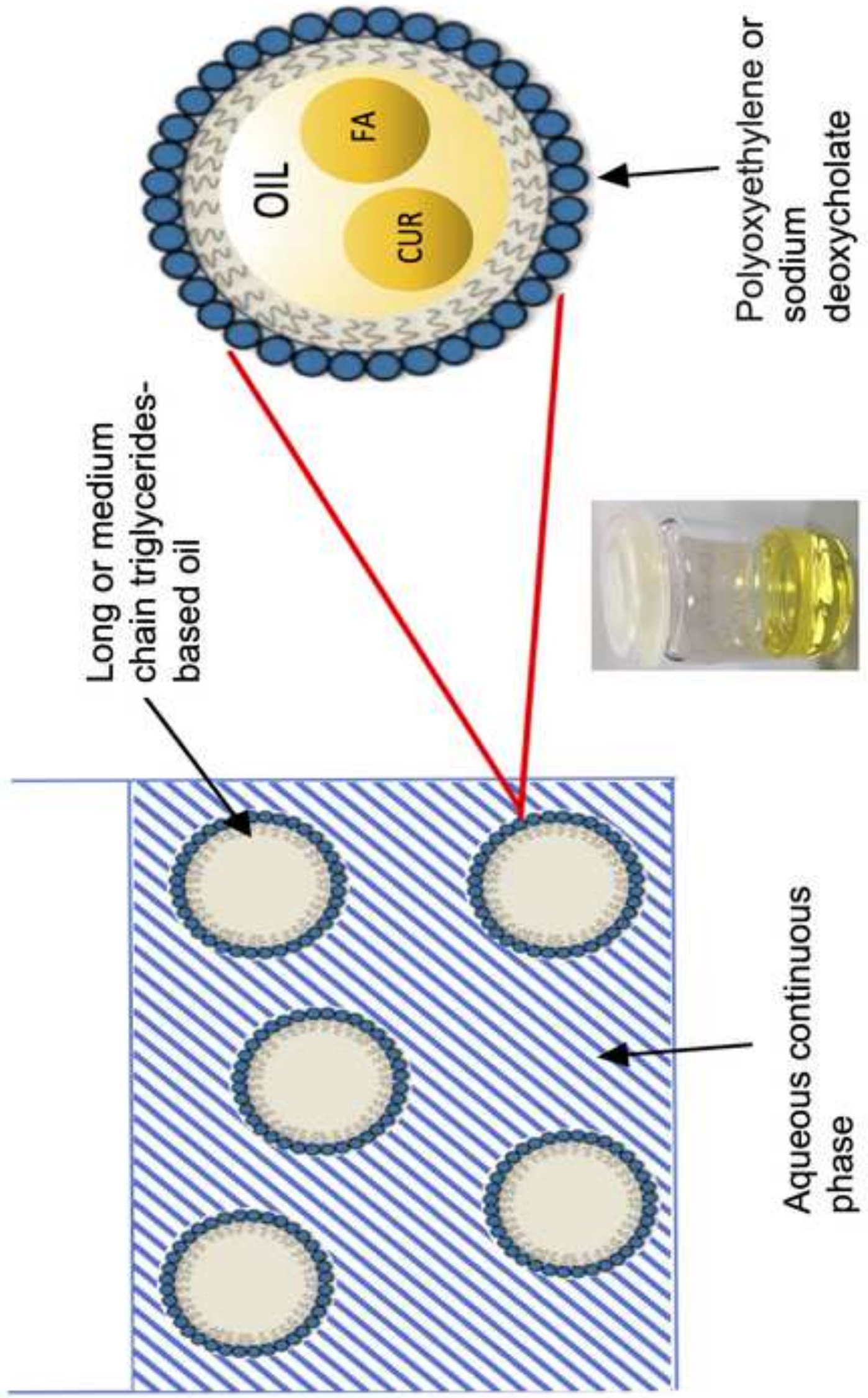


1

2

3

4

5

$23{ }^{*}$ Corresponding author:

24 The School of Pharmacy

25 University of Reading

26 Whiteknights, PO Box 226

27 RG6 6AP

28 Reading, UK

29 T: +44 1183786261

30 h.al-obaidi@reading.ac.uk

31

32 Keywords: curcumin; ferulic acid; microemulsions; degradation; solubility;

33 autoxidation; eutectics 


\section{Abstract}

36 Negligible solubility of curcumin in water combined with rapid degradation have

37 limited its medical applications. In this contribution, eutectic mixture of curcumin with

38 one of its degradation products (ferulic acid) was encapsulated into long and medium

39 chain triglycerides oils and stabilised by the non-ionic surfactant polyoxyethylene

40 oleyl ether Brijß $\mathrm{O} 10\left(\mathrm{C}_{18: 1} \mathrm{E}_{10}\right)$ and bile acid derivative (sodium deoxycholate).

41 Thermal and spectroscopic analysis confirmed the presence of curcumin as solid

42 nanocrystals embedded within the microemulsion droplets. UV-spectroscopy and

43 LC-MS studies of formed microemulsions revealed that degradation of curcumin in

44 water was significantly reduced with more than $83 \%$ of curcumin remained intact

45 after 24 hours. ${ }^{1} \mathrm{H}$ NMR results showed that curcumin remained stable upon heating

46 in the range between $297 \mathrm{~K}-327 \mathrm{~K}$ with no signs of degradation to ferulic acid. The

47 results showed solubility enhancement of curcumin with a range of $3-5 \mathrm{mg} / \mathrm{mL}$. NF-

$48 \quad \mathrm{~KB}$ reporter cell assay revealed low cytotoxicity and three folds stronger anti-

49 inflammatory potential compared to curcumin. Microemulsions remained stable for

50 over 12 months with extended stability of formulations that contained

51 curcumin/ferulic acid in glyceryl tricaprate based microemulsions. Overall these

52 results suggest novel role for particle engineering via using ferulic acid as stability

53 and activity enhancer for curcumin through free radicals scavenging mechanism. 


\section{1. Introduction}

71 Curcumin (CUR) is the most pharmacologically active component in turmeric, which

72 gives rise to its broad spectrum of clinical applications. It has been shown as

73 potential treatment for cancer, Alzheimer's disease and ulcerative colitis [1-3]. A

74 proposed mechanism of action is CUR's role in $\mathrm{O}_{2}$ scavenging [4], NF-kB

75 suppression and downregulation of interleukins and other inflammatory cytokines [5].

76 CUR is also known to prevent tumour growth via inhibition of DNA binding to

77 polycyclic hydrocarbon metabolites [6]. Despite these beneficial properties, CUR has two major disadvantages, limiting its exploitation as a therapeutic agent. Firstly, CUR is practically insoluble in aqueous media, hindering its absorption. As a hydrophobic polyphenol, curcumin is classified based on the biopharmaceutical classification system (BCS) as a class IV molecule due to its low aqueous solubility and its poor penetration through the intestinal wall [7-9]. Secondly, CUR degrades rapidly at physiological conditions, leading to less pharmacologically active metabolites. In neutral or slightly alkaline conditions, CUR degrades by autoxidation and reaction with molecular oxygen to form bicyclopentadione [10]. Other minor degradation products due to alkaline hydrolysis and molecular cleavage are vanillin, ferulic acid and ferulolymethane $[9,11]$. Photodegradation can also occur leading to formation of vanillic and ferulic acid (FA) [11]. Overall, CUR degrades rapidly at physiological conditions, leading to approximately $10 \%$ remaining after 30 minutes [12]. Low solubility is also associated with accumulation of CUR in intestinal wall causing extensive epithelial metabolism [8].

There have been different attempts to improve CUR solubility that were based on use of cyclodextrins, solid dispersions and microemulsions [9, 13-15]. Other examples of previous attempts include solid lipid nanoparticles, transfersomes and liposomes [16-18]. While the benefits of these approaches have been

97 demonstrated, the clinical use of CUR remains limited due to chemical degradation

98 as was previously shown in previous studies $[19,20]$. Hence in this study we 99 present synergistic microemulsion system of triglyceride (oil phase) encapsulating 100 curcumin-ferulic acid eutectic nanocrystals (Figure 1). While this eutectic was 101 reported previously [21], its physiological use remains limited as according to our 102 experiments this eutectic has negligible solubility in water or phosphate buffer. 
103 Ferulic acid (FA) has been shown to exert similar effects to CUR and in some

104 studies, it was suggested that some of the anti-inflammatory effects of CUR were

105 attributed to FA [22]. Recently, it was shown that the adjuvant administration of CUR

106 and FA was effective in counteracting chemoresistance and cisplatin-induced

107 ototoxicity [23]. Exploiting CUR/FA eutectic to prevent degradation of CUR has not

108 been studied hence this will be the focus of this contribution. Since that the oral

109 route represents the major route for drug delivery, the aim of this contribution is to

110 formulate CUR for oral drug delivery.

111

112 We hypothesise that maintaining the eutectic structure as nanocrystals inside the

113 microemulsion droplets/particle can enhance the stability of encapsulated curcumin

114 and achieve a synergistic effect. The use of ferulic acid as a co-former can slow

115 down CUR degradation by scavenging free radicals that initiate autoxidation. The

116 non-ionic surfactant polyoxyethylene oleyl ether $\mathrm{Brij}^{\circledR} \mathrm{O} 10\left(\mathrm{C}_{18: 1} \mathrm{E}_{10}\right)$ and the bile salt

117 derivative sodium deoxycholate (SD) were used to form the microemulsions.

118 Analysis of microemulsions formation was performed to find optimum surfactants

119 system to prepare the microemulsions. It is worth mentioning that the composition of

120 these microemulsions is novel and has not been reported in the literature.

121 Triglycerides are naturally occurring lipids and commonly used as excipients [24].

122 The oil glyceryl tricaprate is 9 carbons medium chain triglyceride while glyceryl

123 trioleate is 17 carbons long chain triglyceride with unsaturation at C9. Apart from the

124 difference in molecular weight, glyceryl trioleate is liquid at room temperature and

125 monounsaturated while glycerol tricaprate is a saturated solid oil at room

126 temperature. To make comparison clearer we will refer to glyceryl trioleate as the

127 liquid oil and for glyceryl tricaprate as the solid oil throughout this research paper.

128 Finally, the impact of the physical state of the oil and microemulsions composition on

129 degradation of curcumin will be discussed.

130

131

132

133

134 2. Material and Methods

135 2.1. Materials 
136 Sodium deoxycholate, trans-ferulic acid 99\%, $\mathrm{C}_{18: 1} \mathrm{E}_{10}$ (Brijß 010$)$ were all obtained

137 from Sigma-Aldrich (Dorset, UK); Glyceryl trioleate and glyceryl tricaprate were

138 obtained from ABITECH Corporation (Janesville, USA); Curcumin (95\% from

139 turmeric rhizome) was obtained from LKT Laboratories (Minnesota, 55130 USA). All

140 the chemicals were used as received. Curcumin was protected from

141 photodegradation using aluminium foil and amber bottles and stored in dark

142 conditions.

143

144 2.2. Pseudo-ternary phase diagrams and phase inversion temperature

145 determination

146 The samples were prepared using glyceryl trioleate or glyceryl tricaprate, surfactant

147 combinations of sodium deoxycholate and/or Brijß 010 and deionised water as the

148 aqueous phase. Different surfactant ratios were explored for all surfactants, of Brijß

149010 to sodium deoxycholate of $1: 1,7: 3$ and 9:1. The oil was added at a weight ratio

150 range between $1-25 \% \mathrm{w} / \mathrm{w}$ and surfactant at a range of $1-24 \% \mathrm{w} / \mathrm{w}$. Systems were

151 classified as a microemulsion, nanoemulsions, emulsion, gel or two-phase system.

152 Pseudo ternary phase diagrams were then generated using XL STAT. This allowed

153 the microemulsion region to be identified and separated from other forms

154 (emulsions, two phase systems, gels or nanoemulsions) in order to perform further

155 analysis. All formulations were made up to $2 \mathrm{~g}$ by mixing the oil with deionised

156 water, sodium deoxycholate, and Brij® O10. Mixtures containing Brijß $\mathrm{O} 10$ were

157 stirred using a magnetic stirrer (Stuart US152 Hot Plate \& Stirrer, UK) in a water bath

158 set at a temperature between $70-75^{\circ} \mathrm{C}$ for $10-15$ minutes. They were then vigorously

159 stirred until reaching room temperature $\left(22^{\circ} \mathrm{C} \pm 2{ }^{\circ} \mathrm{C}\right)$. All samples were monitored

160 regularly for signs of phase separation for a total period of one month. Based upon

161 appearance, samples were categorized as isotropic $\phi_{i}$ or milky/cloudy $\phi_{m}$.

\section{2.3. Preparation of curcumin eutectic mixtures}

164 Eutectic mixtures were made based on variable stoichiometric ratios. The eutectic

165 mixtures were produced using neat solid-state grinding for 15 minutes and

166 mechanochemical activation using a ball mill (Retsch) with frequency of $25 \mathrm{~Hz}$ and

167 milling time of 10 minutes. The mixtures were co-ground, then placed in a grinding

168 jar with a grinding ball using the same stoichiometric ratios of CUR and FA. The 
169

170

171

172

173

174

175

176

177

178

179

180

181

182

183

184

185

186

187

188

189

190

191

192

193

194

195

196

197

198

199

200

201

resultant powders were assessed using thermal analysis, FTIR and x-ray powder diffraction to confirm crystalline structure.

\subsection{Differential scanning calorimetry of microemulsions with eutectic mixtures}

Thermal analysis of the oils and liquid crystals that were made was performed using differential scanning calorimetry (DSC Q2000, TA instruments, UK). Each sample was placed in a crimped aluminium pan before being hermetically sealed. A typical thermogram was obtained by initially allowing the sample to equilibrate at $-50{ }^{\circ} \mathrm{C}$ for 5 minutes. Samples were then heated up to $50{ }^{\circ} \mathrm{C}$ at a heating rate of $10^{\circ} \mathrm{C} / \mathrm{min}$. All samples were purged with nitrogen gas flowing at $50 \mathrm{~mL} / \mathrm{min}$. The thermograms that were produced were then analysed to find the melting points and enthalpy values using Universal Analysis 2000 (TA instruments). All samples were repeated in triplicate.

\subsection{Solubility measurements of curcumin}

CUR and CUR/FA eutectic mixtures were individually added to $1 \mathrm{~mL}$ of microemulsion of desired composition. The samples were then mixed on a rotary mixer for a total period of 72 hours whilst covered with aluminium foil to protect from light. Samples were centrifuged for 5 minutes (Sanyo MSE Micro Centaur, UK) at $13,000 \mathrm{rpm}$ to remove undissolved curcumin. $100 \mu \mathrm{L}$ of each supernatant was then removed and diluted up to $10 \mathrm{~mL}$ with propan-2-ol. A UV-vis measurement of the diluted supernatant was carried out at wavelength of $429 \mathrm{~nm}$.

\subsection{Stability studies using photospectroscopy}

$1 \mathrm{~g}$ of each microemulsion was diluted by a factor of 5 using phosphate buffer $(\mathrm{pH}$ 7.5), and then $1 \mathrm{~mL}$ was transferred to three separate centrifuge tubes that contained CUR or eutectic mixtures. All the tubes with samples were placed onto a rotary mixer with fixed mixing speed for 24 hours and light protected using aluminum foil. Samples were then centrifuged for 5 minutes at 13,000 rpm (Sanyo MSE Micro Centaur, UK). $100 \mu \mathrm{L}$ of the supernatant was taken and diluted to $25 \mathrm{~mL}$ with propan-2-ol. Absorbance was determined at a wavelength of $429 \mathrm{~nm}$ using Varian Cary Bio (Agilent, USA). Absorbance of the same sample was re-measured over 21 
202

203

204

205

206

207

208

209

210

211

212

213

214

215

216

217

218

219

220

221

222

223

224

225

226

227

228

229

230

231

233

234

232 mobile phase. $2 \mu \mathrm{L}$ was injected of circa $50 \mu \mathrm{g} / \mathrm{mL}$ samples that had been made up 235 being directed into the Orbitrap detector scanning in the range $80-2000 \mathrm{~m} / \mathrm{z}$.

days to study overall stability. Degradation experiments were carried out via adding $30 \mu \mathrm{L}$ of the CUR solution (methanol) to $3 \mathrm{~mL}$ of buffer ( $\mathrm{pH} 7.5$ ) in order to maintain the CUR concentration difference between initial concentration of $4 \mathrm{mM}$ to final concentration of $40 \mu \mathrm{M}$.

\section{7. ${ }^{1} \mathrm{H}$ and ${ }^{13} \mathrm{C}$ NMR studies}

${ }^{1} \mathrm{H}$ NMR spectra were recorded on a Bruker Avance III spectrometer (Bruker, Germany) operating at Larmor frequency of $500 \mathrm{MHz}$ (11.75T) using standard Bruker zg30 and noesypr1d pulse sequences. 1D NOESY allowed presaturation of the water signal during the relaxation delay (RD) which improved signal-to-noise ratio of the spectra. The $90^{\circ}$ pulse was $10.8 \mu \mathrm{s}$ at the power level of $17.22 \mathrm{~W}$. The mixing time (tm) was set to $0.05 \mathrm{~s}$. Between 32 and 64 transients were recorded with relaxation delay of 1 and $2 \mathrm{~s}$ respectively for zg30 and noesypr $1 \mathrm{~d}$ and averaged into each spectrum. All spectra were referenced using TSP signal at $0 \mathrm{ppm}$.

The temperature of the sample was changed in the range between 297 and $327 \mathrm{~K}$ in $5 \mathrm{~K}$ steps and controlled with $+/-1 \mathrm{~K}$ error. Once desired temperature was reached each temperature point was equilibrated for $10 \mathrm{~min}$ before acquiring the spectrum. The confirmatory 2D HMBC spectrum was recorded on the $500 \mathrm{MHz}$ spectrometer with ${ }^{1} \mathrm{H}$ parameters optimised as above and the ${ }^{13} \mathrm{C}$ parameters as follows: Larmor frequency of $125.78 \mathrm{MHz}$; the $90^{\circ}$ pulse was $8.25 \mu$ s at the power level of $99.61 \mathrm{~W}$. The spectral widths were $2000 \mathrm{~Hz}$ and $15092.9 \mathrm{~Hz}$ in the direct and indirect dimensions respectively. 2048 and 256 FID points were recorded in the direct and indirect dimension, respectively. 256 single transients were averaged into each of the indirect dimension's points.

\subsection{LC-MS studies}

The analysis was performed using a Thermo Accela HPLC connected to an LTQOrbitrap XL (Thermo Fisher Scientific Inc., San Jose, CA). Chromatographic separations were carried out by means of a Thermo Hypersil Gold 50 x 2.1 mm C18, pore size $175 \AA$, particle size $1.9 \mu \mathrm{M}$ column maintained at $30^{\circ} \mathrm{C}$ and a methanol

233 in methanol. The gradient was isocratic with a flow rate of $200 \mu \mathrm{L} / \mathrm{min}$. The LC-MS 34 was performed with a run time of $10 \mathrm{~min}$. Positive ion mode was employed with ions 
Resolution was set at $15 \mathrm{k}$ and phthalate (413.266230) was used as a lock-mass.

237 Thermo Xcalibur software was used both for acquisition and for data analysis.

238 Extracted ion chromatograms were generated for the protonated and sodiated

239 species of CUR and FA. These were 369.1333, 391.1152 and 195.0652, 217.0471,

240 respectively. Mass tolerance was set at 5 ppm and smoothing, and baseline

241 subtraction was applied.

242

243

\subsection{Dynamic Light Scattering (DLS) and zeta potential measurements}

244 Samples were diluted to $1 \mathrm{~mL}$ with ultra-pure water and transferred to a glass cuvette and analyzed using a Zetasizer (Malvern Zetasizer nano series, UK). The refractive index and viscosity of distilled water were used as reference values. A series of dilutions were made to ensure consistent scattering and to avoid multiple scattering. The temperature was set at $25{ }^{\circ} \mathrm{C}$. Each sample was allowed to equilibrate for 60 seconds and a total of 12 runs per measurement were used with 10 seconds per run. Z-average diameter in nm obtained represented the particle/ droplet size of the samples.

\subsection{X-ray powder diffraction measurements}

254 The polymorphic nature of the formed samples was studies using $x$-ray powder 255 diffraction (XRPD). All samples were scanned using a Bruker D8 advance X-ray 256 diffractometer (Bruker AXS GmbH, Germany) which is a Cu-source, theta- theta 257 diffractometer equipped with a Lynx eye position sensitive detector. It was operated 258 at $40 \mathrm{kV}$ generator voltage and $40 \mathrm{~mA}$ generator current. The samples were 259 analysed using DFFRAC plus XRD commander software (Bruker AXS GmbH, 260 Germany) with a $2 \theta$ range of $5-45^{\circ}$, a step size of $0.02^{\circ}$ and time per step of $1.33 \mathrm{~s}$.

\section{2.11. Cryogenic-Scanning Electron Microscopy (Cryo-SEM)}

263 A cryo-SEM was used to visualise the samples in a frozen hydrated state. A rivet 264 was fitted to a cryo stub and a small amount of the samples were deposited onto the 265 rivet and another rivet was placed on top. The cryo stub was secured to a specimen 266 shuttle which was plunged into nitrogen slush at $-210^{\circ} \mathrm{C}$ and transferred under

267 vacuum to the Quorum PP2000T cryo-SEM preparation chamber (Quorum

268 Technologies Ltd, United Kingdom) at $-190^{\circ} \mathrm{C}$. The sample was then sublimed at $26990^{\circ} \mathrm{C}$ for 15 minutes. The temperature of the preparation chamber was then lowered 
270

271

272

273

274

275

276

277

278

279

280

281

282

283

284

285

286

to $-135^{\circ} \mathrm{C}$ and the sample was sputter coated with a thin layer of platinum for 80 seconds at $9-10 \mathrm{~mA}$. The sample was transferred to the FEI Quanta 600 FEG scanning electron microscope (FEI, Eindhoven, Neverlands) in high vacuum mode at $-135^{\circ} \mathrm{C}$ and the images were recorded at an accelerating voltage of $20 \mathrm{kV}$.

\subsection{Cultivation of U251 Cells}

Human glioblastoma cell line U251 (Cell Line Service, Eppelheim, Germany) were cultivated in Dulbecco's Modified Eagle's Medium (DMEM)-High glucose (SigmaAldrich, Gillingham, United Kingdom) supplemented with 1\% L-glutamine (200 mM, Sigma-Aldrich) and 10\% heat-inactivated fetal bovine serum (Sigma-Aldrich, Gillingham, United Kingdom). Human glioblastoma cell line U251-NF-KB-GFP-LUC reporter cells were generated previously [25] and were cultivated in normal cultivation media supplemented with $5 \mu \mathrm{g} / \mathrm{mL}$ puromycin (Apollo Scientific, Stockport, U.K.). All cells were maintained in a humidified incubator at $37^{\circ} \mathrm{C}$ and $5 \%$ (v:v) $\mathrm{CO}_{2}$. Early passage U251 cells (passage 3-20) were used for all experiments. U251 cells are well established model cells that are used for drug screen and signal transduction research in cancer cells [26-28]. Importantly, this cell line is also widely used to study the impact of curcumin on cancer cells including studying of pro- and anti-inflammatory signal transduction pathways [29-31]. In this context, NF-kappaB is not crucially involved in regulation of inflammation but also regulates cancer cell proliferation and migration and plays a pivotal role in drug resistance [32, 33]. Therefore, assessing NF-kappaB activity in U251 cells represents a widely used and state-of-the-art approach to study the impact of anti-inflammatory drugs on various cancer cells.

\subsection{Cell Viability Assay}

Cell viability assays were performed using the Cell Proliferation Kit II (Sigma-Aldrich) according to manufacturer's recommendations. Briefly, $5 \times 10^{3} \mathrm{U} 251$ cells per well were seeded in 96-well plates for 24 hours to reach $50 \%$ confluency. Subsequently, the medium was changed to the cultivation medium with the respective treatment and XTT assay was performed after 48 hours of incubation. Absorbance was measured using Spectra Max iD3 plate reader (Molecular Devices, Wokingham, United Kingdom) at an excitation wavelength of $490 \mathrm{~nm}$ and a reference wavelength of $650 \mathrm{~nm}$. 


\subsection{Luciferase Assay}

306 U251-NFKB-GFP-LUC cells were seeded in 24-well plates in normal cultivation

307 medium prior to two hours starvation in FBS-free medium and subsequently exposed

308 to $10 \mu \mathrm{M}$ of the respective curcumin formulation, $10 \mathrm{ng} / \mathrm{mL}$ tumour necrosis factor-

309 alpha (TNF-a, (PeproTech), TNF-a /curcumin, ultrapure E. coli-derived

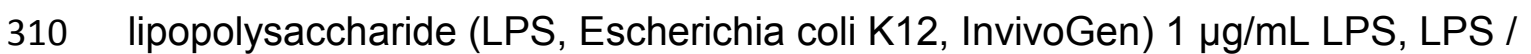

311 curcumin, TNF-a/Bay-11-7082, or LPS/Bay-11-7082, respectively.

312 Cells were lysed after 48 hours of incubation and the luciferase bioluminescence

313 was assessed using the Luciferase Assay System (Promega, Southampton, United

314 Kingdom) and a Spectra Max iD3 plate reader.

\subsection{Statistical analysis}

317 GraphPad Prism 5 was used to perform statistical analysis. Student's t-test (two-

318 tailed, 95\% confidence interval) or one-way ANOVA with Bonferroni's Multiple

319 Comparison Test (95\% confidence interval) was applied, where appropriate. Data

320 from at least three independent experiments was collected and $P$ value $<0.05$ was

321 considered as significant.

\section{3. Results and discussion}

\section{3.1. Formation regions of microemulsions based on pseudo-ternary phase}

\section{5 diagrams and particle/ droplet size analysis}

326 Formation of small droplets in the dispersed phase is associated with a large

327 reduction of surface tension and therefore stable microemulsions are formed [34].

328 Depending on the composition of surfactants, other less stable intermediate

329 structures such as emulsions nanoemulsions can also form. The distinction between

330 the different structures was made based on visual inspection and droplets/particles

331 size measurements. To form the microemulsions, three ratios of Brij $® 010$ to

332 sodium deoxycholate (SD) were prepared, namely $1: 1,7: 3$ and $9: 1$. In addition,

333 microemulsions were formed using $\mathrm{Brij} \circledast \mathrm{O} 10$ alone. These ratios were guided by

334 preliminary studies where it was not possible to form emulsions or microemulsions 
using molar ratios Brij® 010 to SD below 1:1. Likewise, it was not possible to form microemulsions when sodium deoxycholate was used as the sole surfactant.

Pseudo-ternary phase diagrams were used to map the area of existence for amphiphilic association structures (i.e. emulsion, microemulsion, nanoemulsion). The area of existence was divided into isotropic, transparent or opalescent samples $\left(\phi_{i}\right)$ and cloudy/milky samples $\left(\phi_{m}\right)$. Samples that were in the $\phi_{i}$ region were microemulsions or nanoemulsions while samples that were in the $\phi_{m}$ region were considered emulsions. Results showed that samples prepared using Brijß 010 with either solid-based glyceryl tricaprate or liquid-based glyceryl trioleate lead to formation of microemulsions (Figure 2). The general trend showed that the solid oil formed more microemulsions when compared with the liquid oil. The use of Brij $\AA$ O10 has generated more microemulsions while when Brijß 010 was mixed with SD, the number of formed microemulsions was lowered. This trend could be clearly seen when using ratios of Brijß 010:SD <9:1 as at these ratios only emulsions were formed. At a molar ratio of Brijß 010:SD of 9:1, a mixture of emulsions, nanoemulsions and microemulsions was formed.

Particle/droplet size analysis of microemulsions showed similar trends to those observed in the pseudo-ternary phase diagrams (Figure 3). As can be seen, the size of the microemulsions droplets increased with reducing the surfactant ratio with the size varied from $12.0 \pm 0.04 \mathrm{~nm}$ to $23.9 \pm 0.1 \mathrm{~nm}$, in proportion to reduced ratio of Brijß 010 from $24 \%$ to $15 \%$ and increased ratio of oil from $5 \%$ to $10 \%$. The smallest particle/ droplet size was found for samples prepared using $5 \%$ glyceryl tricaprate (solid oil) and 24\% Brij ${ }^{\circledR} \mathrm{O} 10(12.0 \pm 0.036 \mathrm{~nm}$ ) which was statistically similar to the size of microemulsions droplets formed using $5 \%$ glyceryl trioleate (liquid oil) and $24 \%$ Brijß O10 $(12.4 \pm 0.148 \mathrm{~nm})(p<0.05)$. Overall, a statistical difference in particle/ droplet size was found between microemulsions formed from the solid oil when compared with microemulsions formed form the liquid oil (Figure 3). The statistical analysis was based on one-way analysis of variance (ANOVA) ( $p$-value $<0.05$ ) (Figure 3). This aligns with the above findings that showed that the solid oil formed more microemulsions compared to the liquid oil (Figure 2). The zeta potential measurements showed that the particles/ droplets exhibited negative values (Figure 3 ) with values increasing in proportion to the surfactant's ratio (-63 to $-50 \mathrm{mv}$ ). 
369 These values agreed with previously reported values for Brijß 010 emulsions [35].

370 The relatively high zeta potential values can have dramatic impact on the physical

371 stability of the formed microemulsions. The resulting repulsion due to the associated

372 surface charges can be detrimental for maintaining the droplets/ particles segregated

373 and prevent possible aggregation and phase separation.

\subsection{Incorporation of CUR and CUR-FA eutectic mixtures}

376 The CUR/FA eutectic mixtures were prepared using mechanochemical activation

377 using different stochiometric ratios (1:1, 1:2, 1:3). Formation of the eutectic mixtures

378 was confirmed using thermal analysis and XRPD (Figure 4). CUR:FA (1:1) showed a

379 single melting point indicating the formation of single phase, hence this composition

380 was used throughout this study. The other ratios (1:2 and 1:3) showed a shoulder

381 indicating that the mixtures contained eutectic mixtures in addition to pure

382 components. Lack of exothermic recrystallization peak suggests that the formed

383 structures are eutectic mixtures rather than corresponding co-crystals [21, 36].

384 Confirmation of formation of eutectic mixture was further confirmed using x-ray

385 powder diffraction by which no new peaks were detected in agreement with thermal

386 analysis results (Figure 4).

387 CUR/FA eutectic mixtures were incorporated into the microemulsions and the final

388 particles/ droplet were assessed using thermal analysis, particle/ droplet size and

389 solubility measurements. As can be seen in Figure 5, the melting peak onset of the

390 solid oil (glyceryl tricaprate) was observed at around $29^{\circ} \mathrm{C}$ while the sharp melting

391 endotherm at $0^{\circ} \mathrm{C}$ refers to melting of ice and the melting at $-20^{\circ} \mathrm{C}$ refers to melting

392 of the surfactant Brij® O10. The corresponding microemulsion showed slightly

393 lower melting of the oil at around $25^{\circ} \mathrm{C}$ (marked with number 1 in Figure 5) which

394 indicates that the oil phase was solid/semi solid at room temperature. This is

395 important finding as it indicates that the drug or eutectic mixtures are entrapped

396 inside solid nanoparticles rather than liquid droplets which can have significant

397 impact on stability. When CUR was incorporated into the microemulsion, the melting

398 peak of the oil can still be seen at $25^{\circ} \mathrm{C}$. It is interesting to observe that when the

399 eutectic mixture was incorporated into the microemulsion, a new endothermic peak 
400

401

402

403

404

405

406

407

408

409

410

411

412

413

414

415

416

417

418

419

420

421

422

423

424

425

426

427

428

429

430

431

432

could be observed at $13^{\circ} \mathrm{C}$. We anticipate that the CUR containing microemulsion is partially solid at room temperature while the CUR/FA is predominately liquid (or highly viscous liquid). Similar observation was reported before by which the drug can exist inside solid droplets of nanoemulsions/ microemulsions [37].

The liquid oil showed a melting peak onset at around $-20^{\circ} \mathrm{C}$ preceded by recrystallization peak at $-28^{\circ} \mathrm{C}$. It was not possible to observe a melting peak of the liquid oil when CUR or CUR/FA were incorporated. This behavior often reflects favorable interactions preventing recrystallization and associated melt. The thermogram of the liquid oil microemulsions showed the lack of an endotherm indicating that the oil phase was completely in the liquid state. It is expected that at room temperature, the kinetic energy would have been sufficient to weaken any intermolecular interactions. It is anticipated that the impact of thermal events happening in the solid oil will have greater impact than the liquid oil on the physicochemical properties of CUR and CUR/FA. As mentioned above, it was possible to observe the endothermic peak associated with the melt of the solid oil at approximately $25^{\circ} \mathrm{C}$. This was shifted to $13^{\circ} \mathrm{C}$ upon the addition of the CUR/FA eutectic mixtures. Such change indicates structural changes and potentially interactions between the oil and CUR/FA. More importantly, a solid core could limit mobility and enhance stability. Similar trend was observed before for solid lipid nanoparticles with melting endotherms reflected in different solubility of the drug [37].

As was seen above, the melting endotherm for the oil seemed to change when either CUR or CUR/FA were added. In order to understand the reason for this phenomenon, it became important to study CUR-oil and CUR/FA-oil thermodynamics of mixing. To achieve this, CUR or CUR/FA were dissolved into the solid oil and thermal events were then characterized. As can be seen in Figure 6, the melting peak onset of CUR/FA can be seen at $149^{\circ} \mathrm{C}$. The melting endotherms of the pure CUR and pure FA can be seen at 177.6 and $173^{\circ} \mathrm{C}$, respectively. Once the oil melted, it did not show any endothermic peaks that could indicate evaporation or degradation within measured temperature range. There was a single endothermic peak at around $140{ }^{\circ} \mathrm{C}$ which is significantly lower than the melting endotherm of the CUR/FA. We anticipate that this is the eutectic CUR/FA however this may correspond to co-crystals formation. Examination of the XRPD peaks were not 
conclusive as because of the low intensity of CUR/FA compared to the oil. Nevertheless, observing the CUR/FA peak within the oil signifies that the CUR/FA mixture maintained its crystalline structure inside the oil phase instead of separating as separate FA or CUR crystals. The fact that microemulsions remained visually clear, suggest that CUR/FA existed as nanocrystals inside the oil particles/ droplets. The solid oil melted at $25^{\circ}$ showing two polymorphic transitions at $11^{\circ} \mathrm{C}$ and $-7^{\circ} \mathrm{C}$ which were not affected by incorporation of CUR or CUR/FA eutectic mixtures. The cooling rate was controlled at $10{ }^{\circ} \mathrm{C} / \mathrm{min}$ while a fast cooling cycle was used for microemulsions to avoid structural changes in the microemulsion system. Hence, these polymorphic changes were not seen in Figure 5.

Incorporation of the CUR or CUR/FA eutectic mixtures resulted in changes in particle/ droplet size of the microemulsions. Particle/ droplet size of microemulsions incorporating CUR or with CUR/FA was statistically different $(p<0.05)$ to microemulsions (Figure 7). As shown above, using a combination of SD and Brijß $\mathrm{O} 10$ resulted in lower number of formed microemulsions than when using Brijß 010 . Upon incorporation of CUR or CUR/FA, the particle size has grown to a larger extent in microemulsions prepared using the surfactants mixture than $\mathrm{Brij} \circledast 010$. This trend was more noticeable when the liquid oil was used to from the microemulsions resulting in approximate difference of $10 \mathrm{~nm}$. It is interesting to observe that there was a minimum impact of CUR/FA inclusion on the microemulsions prepared using the solid oil. In fact, when the mixture of SD and Brijß 010 was used, the size of the microemulsions particles/ droplets decreased by 2-3 nm. These results mirror the thermal analysis findings observed above by which the solid oil based microemulsions maintained smaller particle/ droplet size than the liquid oil based microemulsions. Existence of the CUR or CUR/FA as solid entities within the physically stable droplets can prevent CUR degradation and precipitation when exposed to aqueous environment. Cryo- SEM was used to examine the structure of the microemulsions to assess if the increased size was due to aggregation of the formed particles. As can be seen in Figure 7, formed microemulsions maintained their segregated structures with uniform size distribution. The continuous globular structures represent frozen oil droplets enwrapped within the Brijß 010 network. 
465

466

467

468

469

470

471

472

473

474

475

476

477

478

479

480

481

482

483

484

485

486

487

488

489

490

491

492

493

494

495

496

88

These results support the thermal analysis findings in that CUR as well as CUR/FA existed as nanocrystals inside the oil phase and in equilibrium with dissolved CUR/FA.

\subsection{Enhanced solubility of curcumin in Brij® 010/SD microemulsions}

As shown in Figure 8, microemulsions showed significantly higher $(p<0.05)$ solubility of CUR alone when compared to CUR/FA eutectic mixtures in both solid glyceryl tricaprate and liquid glyceryl trioleate based microemulsions. For instance, the highest solubility was $5.56 \pm 0.13 \mathrm{mg} / \mathrm{mL}$ for microemulsions prepared with $24 \%$ Brij $囚 010$ and $10 \%$ glyceryl tricaprate for CUR. This was significantly higher $(p<0.05)$ than $4.84 \pm 0.08 \mathrm{mg} / \mathrm{mL}$ and $4.85 \pm 0.14 \mathrm{mg} / \mathrm{mL}$ for the CUR/FA eutectic mixtures in solid and liquid oil based microemulsions, respectively. Overall, incorporation of CUR into microemulsions contributed to a solubility of CUR ranging from 3.71-5.56 mg/mL, whilst 3.14-4.89 mg/mL was the range of those with the addition of CUR/FA eutectic mixtures. The reduced solubility of CUR when the CUR/FA was added suggests that FA is localized within the oil phase leading to lower solubility of CUR. If FA was dissolved within the continuous aqueous phase, then a reduction in solubility of CUR would not be affected. This additional occupancy of FA lead to increased size of microemulsion particles/droplet as observed above. The aqueous solubility of CUR was found to be $0.16 \mu \mathrm{g} / \mathrm{mL}$ while the solubility of CUR from the eutectic mixture was $0.06 \mu \mathrm{g} / \mathrm{mL} \mu \mathrm{g}$ which suggests that CUR/FA eutectic mixtures were present as practically insoluble nanocrystals.

Interestingly, referring to Figure 9, microemulsions prepared using Brijß $\mathrm{O} 10$ as the only surfactant showed greater solubility of CUR when compared with the mixture of Brijß 010 and sodium deoxycholate in both solid and liquid oil based microemulsions. For example, with the combination of $24 \%$ surfactant and $5 \%$ oil, sample prepared with single surfactant had a solubility of $5.32 \pm 0.14 \mathrm{mg} / \mathrm{mL}$ which was significantly higher $(p<0.05)$ than that of $3.90 \pm 0.1 \mathrm{mg} / \mathrm{mL}$ with surfactant mixture using glyceryl tricaprate as the oil; whilst solubility of $5.39 \pm 0.12 \mathrm{mg} / \mathrm{mL}$ and $3.83 \pm 0.27 \mathrm{mg} / \mathrm{mL}$ were obtained for microemulsion prepared using glyceryl trioleate 
497 with single surfactant and surfactant mixture, respectively. However, the differences

498 in CUR solubility between the glyceryl tricaprate (solid based) and glyceryl trioleate

499 (liquid based) oils was not significant ( $p>0.05$ ), irrespective of the use of single

500 surfactant or surfactant mixture. The lower solubility of CUR in the SD containing

501 microemulsions suggest unfavorable head groups interaction with Brijß 010 causing

502 CUR to localize deeper inside the oil droplets. The core of the microemulsion

503 droplets comprises entirely of the oil enclosed by the oleyl hydrocarbons of the

504 surfactant molecules and lastly by a mantle of ethylene oxide chains, leading to

505 enhancement in hydration as the distance from the core increases [38]. Solubility of

506 CUR was monitored regularly to ensure that CUR remained physically stable. The

507 samples were stored at room temperature and dark conditions as CUR can undergo

508 photodegradation [39]. The data in Figure 9 show that CUR had similar solubility in

509 all samples prepared after 21 days of preparation. There was no statistical

510 difference $(p<0.05)$ in solubility of CUR in freshly prepared samples and samples

511 that were prepared 21 days after solubility measurement. Hence, prepared

512 microemulsions were physically stable; chemical degradation can still occur

513 therefore it was investigated as shown below.

515 3.4. Assessment of CUR degradation using photospectroscopy, mass

516 spectrometry and temperature controlled ${ }^{1} \mathrm{H}$ NMR

517 While solubility enhancement of CUR was previously reported [40, 41], our study

518 provides a detailed analysis of the stability of CUR while being dissolved in the oil

519 phase as well as after being released from the vehicles. It has been reported before

520 that a possible degradation pathway for CUR is autoxidation and solvolysis [10, 42].

521 To simulate these degradation conditions, phosphate buffer mixed with methanol

522 was used for diluting the samples while CUR concentration was checked at regular

523 intervals for a total duration of 24 hours. UV spectrophotometer was used to

524 monitor CUR peak at wavelength of $429 \mathrm{~nm}$. Within this region there are no

525 overlapping peaks with degradation products nor from FA. Measurements were

526 performed at room temperature and $37^{\circ} \mathrm{C}$ using phosphate buffer to dilute the

527 samples. As can be seen in Figure 10, CUR degraded within 20 minutes to less

528 than $50 \%$ of its original concentration. Autoxidation has been shown to be major 
pathway for CUR via phenoxy free radical formation and propagation through interaction with molecular oxygen $[10,43]$. In order to minimize autoxidation when dissolving CUR into the phosphate buffer, degassing was used to remove dissolved oxygen before adding CUR to the buffer. As can be seen, initial rate of CUR degradation was slowed, however faster degradation kinetics occurred after 15 minutes. These results confirm that the presence of oxygen accelerated the degradation of CUR. Furthermore, addition of DMSO to the phosphate buffer showed slower degradation kinetics which is likely because the organic solvent reduces the amount of free ${ }^{\circ} \mathrm{OH}$. Interestingly, dissolving CUR and FA slowed down degradation in a similar pattern to inclusion of DMSO (Figure 10). This confirms the suggestion that $\mathrm{FA}$ can scavenge free ${ }^{\circ} \mathrm{OH}$ which initiate the autoxidation process. Inclusion of FA did not completely stop the process which could be attributed to partial scavenging and alkaline hydrolysis at the ketone moiety.

To simulate degradation at ambient conditions, degradation of CUR was studied at room temperature. As can be seen in Figure 11, initial steady reduction in CUR was observed in all samples (microemulsions and pure CUR). Liquid oil based microemulsions showed a similar trend to solid oil based microemulsions, but CUR degraded at a faster rate in liquid oil based microemulsions. There was a positive impact for the inclusion of FA reflected by higher amount of remaining CUR, where the range of percentage remaining was $70.20 \pm 0.70-83.26 \pm 1.24 \%$ when CUR/FA eutectic mixtures were incorporated. This is in contrast to $55.42 \pm 0.82 \%-77.05 \pm$ $0.76 \%$ when CUR alone was added to the microemulsions. Overall, $17 \%-45 \%$ of incorporated CUR degraded after exposure to phosphate buffer at $\mathrm{pH} 7.5$, while the percentage of degradation of CUR without incorporation was $63 \%$, indicating that incorporating CUR into microemulsions significantly improved the chemical stability of CUR $(p<0.05)$. Solid oil based microemulsions prepared using Brijß 010 embedding CUR/FA eutectic mixtures showed best stability of CUR. This could be seen as $37.48 \pm 2.48 \%$ of CUR alone remained while the microemulsions embedding the eutectic mixtures had significantly higher percentage $(p<0.05)$ of $83.26 \pm 1.24 \%$, showing a marked difference of $45.78 \%$. 
562 The mixture of Brijß 010 and SD showed improved stability of CUR in both liquid

563 and solid oils when CUR/FA eutectic mixtures were used. Similar effect was seen for

564 Brijß 010 and SD mixture with CUR/FA. SD has been recently used as a reducing

565 agent with oxidation happening at the hydroxyl groups to form carbonyl groups [44].

566 This oxidation process was UV induced hence it may contribute to further

567 stabilisation of CUR against photodegradation. This effect was reversed when CUR

568 was incorporated into the microemulsions which we speculate that could be due to

569 induction of alkaline hydrolysis which seemed to be prevented in the presence of

570 partially ionised FA.

572 Mass spectrometry was used to monitor CUR peak using pure water as solvent and

573 compared with methanol CUR solution. As can be seen (Figure 10), CUR peak was

574 detected as 369.1333 and 391.1152 and for FA the peaks were 195.0652 and

575217.0471 (protonated and sodiated species, respectively). It was possible to detect

576 CUR peak in microemulsions with approximate intensity to the CUR peak with the

577 FA eutectic mixtures. The FA peak can also be seen indicating no change in its

578 intensity over time. The focus of this study is the possible conversion of CUR to FA,

579 but our findings showed that CUR does not degrade into FA. While FA could be a

580 minor degradation product [45], its presence improved stability of CUR possibly via

581 scavenging free radicals, which were identified as possible mechanism for CUR

582 degradation [19].

583 As shown above, degradation rate happened significantly faster at $37^{\circ} \mathrm{C}$ compared

584 to ambient temperature $\left(20-22^{\circ} \mathrm{C}\right)$. Heating has been shown to accelerate

585 degradation of CUR to happen within less than ten minutes [42]. To simulate the

586 impact of temperature on degradation, the thermal stability of CUR was assessed

587 using temperature controlled ${ }^{1} \mathrm{H}$ NMR experiment in the biologically relevant

588 temperature range of $297 \mathrm{~K}$ to $327 \mathrm{~K}\left(24\right.$ to $\left.54^{\circ} \mathrm{C}\right)$. CUR in methanol-d4 is present

589 as CUR and demethoxycurcumin in equilibrating keto-enol tautomers. There was

590 clear evidence of that in the HMBC spectrum (data not shown) which agreed with

591 previously published studies [46]. The comparison of ${ }^{1} \mathrm{H}$ spectra in Figure 12 (a) and

592 (b) shows that between 6.4 and 7.8 ppm the observed signals originate

593 predominantly from CUR, not from either the oil or surfactant used. The 
characteristic pattern of CUR signals in methanol-d4 is repeated for CUR in oil/surfactant $/ \mathrm{D}_{2} \mathrm{O}$. However, the chemical shifts, as well as widths of most peaks were affected by the change in neighboring environment, which is likely to be caused by the difference in the solvent. The ${ }^{1} \mathrm{H}$ spectra in Figure 12 (c) shows CUR signals recorded between 297 and 327 K. No significant changes have been observed (only typical temperature shifts) suggesting that the CUR remains stable within this temperature range. Overall, these results confirm findings obtained above showing that CUR did not degrade to FA at least within short time scale and it remained stable when formulated into the microemulsions. The results do not confirm whether autoxidation is happening and whether bicyclopentadione as major degradation product is formed, however these results confirm that increasing temperature did not lead to FA formation. A previous study has shown that CUR undergoes thermal degradation leading to the formation of FA as a major degradation product [47]. Hence, our results suggest that CUR resisted this degradation possibly through physical complexation with FA.

\subsection{CUR shows increased anti-inflammatory potential}

Visual inspection of microemulsions after storage for 12 months showed that glyceryl trioleate (liquid oil) based microemulsions vanished when compared with glyceryl tricaprate (solid oil) based microemulsions (Figure 13). The color intensity of CUR was retained in both solid and liquid oil based microemulsions that contained CUR/FA eutectic mixture signifying the positive effect of including FA in the microemulsions. It is becoming clear that the use of FA to extend the stability of CUR is a successful approach. Incorporation into microemulsions has significantly enhanced CUR solubility to approximately $5 \mathrm{mg} / \mathrm{mL}$ compared to aqueous solubility of $60 \mathrm{ng} / \mathrm{mL}$. The remaining question whether the combination of CUR and FA had any impact on anti-inflammatory potential. In order to validate that the decrease of NF-kappaB signal caused by the CUR formulations is a result of their antiinflammatory activity and not caused by reduced cell viability, XTT assays were performed. U251Cells were exposed to increasing concentrations of CUR followed by measuring the XTT absorbance. Analysis of the data showed no negative impact on cell viability (Supplementary data) at the concentrations used for the assessment 
626 of NF-kappaB activity $(10 \mu \mathrm{M})$. To assess the anti-inflammatory potential of freshly

627 prepared curcumin formulations, a previously developed U251-NF-KB-GFP-LUC

628 reporter system was used [25]. In order to simulate inflammation in vitro and to

629 assess the anti-inflammatory potential of the different CUR formulations, reporter

630 cells were exposed to the well characterised pro-inflammatory molecules TNF- $\alpha$, or

631 LPS or combinations of CUR formulations with TNF- $\alpha$ or LPS. As an additional

632 control, the broad spectrum anti-inflammatory inhibitor Bay-11-7082 was used [48]. A

633 significant up-regulation of the NF-kB-dependent luciferase bioluminescence was

634 observed in both cells exposed to TNF- $\alpha$ and LPS (Figure 13). Notably, all new CUR

635 formulations showed stronger anti-inflammatory action for both stimuli. The effect

636 was three-fold stronger than CUR dissolved in DMSO. The difference among the

637 studied microemulsions was insignificant signifying that CUR had similar activity in

638 all microemulsions. However, CUR/FA containing formulations contained ratio of 1:1

639 hence the effect of CUR was significantly augmented in CUR/FA microemulsions

640 and estimated to be two folds stronger anti-inflammatory potential compared to CUR

641 microemulsions.

642

\section{4. Conclusions}

644 In summary, microemulsions incorporating curcumin and ferulic acid showed

645 improved solubility, stability as well as improved blockage of the cytokine, TNF- $\alpha$.

646 The solubility enhancement was significantly higher than previously reported

647 methods such as incorporation into micellar solutions [49], use of solubilizers [50]

648 and microencapsulation in whey protein isolate [51]. This represents a novel

649 approach to maintain curcumin in its native form and prevent its possible

650 degradation. Assessment of oil droplets size revealed a narrow and consistent size

651 distribution which was affected by the presence of curcumin /ferulic acid. The

652 physical nature of the oil used to prepare the microemulsions had a significant

653 impact on stability as was reflected by solid glyceryl tricaprate based microemulsions

654 compared to the liquid glyceryl trioleate microemulsions. This was attributed to the

655 presence of curcumin /ferulic acid nanocrystals that were shown to exist in the solid

656 oil as indicated by thermal analysis results. NMR and LC-MS studies confirmed that

657 curcumin did not convert into ferulic acid even when exposed to higher

658 temperatures. Overall, these results present a novel solution to overcome poor

659 absorption associated with the use of curcumin. 
Acknowledgements

662 The authors would like to thank the Chemical Analysis Facility at the University of

663 Reading for providing essential access to instruments used in this study.

Declaration of Interest

\section{Author Contributions}

\section{References}

[1] R.E. Carroll, R.V. Benya, D.K. Turgeon, S. Vareed, M. Neuman, L. Rodriguez, M. Kakarala, P.M. Carpenter, C. McLaren, F.L. Meyskens, D.E. Brenner, Phase Ila Clinical Trial of Curcumin for the Prevention of Colorectal Neoplasia, Cancer Prevention Research, 4 (2011) 354.

[2] M. Kanai, Y. Otsuka, K. Otsuka, M. Sato, T. Nishimura, Y. Mori, M. Kawaguchi, E. Hatano, Y. Kodama, S. Matsumoto, Y. Murakami, A. Imaizumi, T. Chiba, J. Nishihira, H. Shibata, A phase I study investigating the safety and pharmacokinetics of highly bioavailable curcumin (Theracurmin ${ }^{\circledR}$ ) in cancer patients, Cancer Chemotherapy and Pharmacology, 71 (2013) 15211530.

685 [3] D. Yanagisawa, N. Shirai, T. Amatsubo, H. Taguchi, K. Hirao, M. Urushitani, S. Morikawa, T. Inubushi, M. Kato, F. Kato, K. Morino, H. Kimura, I. Nakano, C. Yoshida, T. Okada, M. Sano, Y. Wada, K.N. Wada, A. Yamamoto, I. Tooyama, Relationship between the tautomeric structures of curcumin derivatives and their Abeta-binding activities in the context of therapies for Alzheimer's disease, in: Biomaterials, 2010 Elsevier Ltd, Netherlands, 2010, pp. 4179-4185.

689 [4] L. Shen, C.C. Liu, C.Y. An, H.F. Ji, How does curcumin work with poor bioavailability? Clues from experimental and 690 theoretical studies, Sci Rep, 6 (2016) 20872.

691 [5] B.B. Aggarwal, K.B. Harikumar, Potential Therapeutic Effects of Curcumin, the Anti-inflammatory Agent, Against Neurodegenerative, Cardiovascular, Pulmonary, Metabolic, Autoimmune and Neoplastic Diseases, The international journal of biochemistry \& cell biology, 41 (2009) 40-59.

694 [6] M.T. Huang, Z.Y. Wang, C.A. Georgiadis, J.D. Laskin, A.H. Conney, Inhibitory effects of curcumin on tumor initiation by benzo[a]pyrene and 7,12-dimethylbenz[a]anthracene, Carcinogenesis, 13 (1992) 2183-2186.

696 [7] M.K. John, H. Xie, E.C. Bell, D. Liang, Development and pharmacokinetic evaluation of a curcumin co-solvent formulation, 697 Anticancer Res, 33 (2013) 4285-4291.

698 [8] B. Wahlang, Y.B. Pawar, A.K. Bansal, Identification of permeability-related hurdles in oral delivery of curcumin using the 699 Caco-2 cell model, European journal of pharmaceutics and biopharmaceutics : official journal of Arbeitsgemeinschaft fur 700 Pharmazeutische Verfahrenstechnik e.V, 77 (2011) 275-282.

701 [9] H.H. Tonnesen, M. Masson, T. Loftsson, Studies of curcumin and curcuminoids. XXVII. Cyclodextrin complexation: solubility, chemical and photochemical stability, International journal of pharmaceutics, 244 (2002) 127-135. 
[12] Y.J. Wang, M.H. Pan, A.L. Cheng, L.I. Lin, Y.S. Ho, C.Y. Hsieh, J.K. Lin, Stability of curcumin in buffer solutions and characterization of its degradation products, in: J Pharm Biomed Anal, England, 1997, pp. 1867-1876.

708 [13] C.C. Lin, H.Y. Lin, M.H. Chi, C.M. Shen, H.W. Chen, W.J. Yang, M.H. Lee, Preparation of curcumin microemulsions with food-grade soybean oil/lecithin and their cytotoxicity on the HepG2 cell line, Food Chem, 154 (2014) 282-290. [14] C.C. Teixeira, L.M. Mendonca, M.M. Bergamaschi, R.H. Queiroz, G.E. Souza, L.M. Antunes, L.A. Freitas, Microparticles Containing Curcumin Solid Dispersion: Stability, Bioavailability and Anti-Inflammatory Activity, AAPS PharmSciTech, 17 (2016) 252-261.

[15] V. Kakkar, S. Singh, D. Singla, I.P. Kaur, Exploring solid lipid nanoparticles to enhance the oral bioavailability of curcumin, Molecular nutrition \& food research, 55 (2011) 495-503.

[16] S. Huang, J. He, L. Cao, H. Lin, W. Zhang, Q. Zhong, Improved Physicochemical Properties of Curcumin-Loaded Solid Lipid Nanoparticles Stabilized by Sodium Caseinate-Lactose Maillard Conjugate, Journal of agricultural and food chemistry, 68 (2020) 7072-7081.

[17] Y. Chen, Q. Wu, Z. Zhang, L. Yuan, X. Liu, L. Zhou, Preparation of curcumin-loaded liposomes and evaluation of their skin permeation and pharmacodynamics, Molecules, 17 (2012) 5972-5987.

[18] H. Chaudhary, K. Kohli, V. Kumar, Nano-transfersomes as a novel carrier for transdermal delivery, International journal of pharmaceutics, 454 (2013) 367-380.

[19] M. Griesser, V. Pistis, T. Suzuki, N. Tejera, D.A. Pratt, C. Schneider, Autoxidative and cyclooxygenase-2 catalyzed transformation of the dietary chemopreventive agent curcumin, J Biol Chem, 286 (2011) 1114-1124.

[20] B. Zheng, X. Zhang, S. Peng, D. Julian McClements, Impact of curcumin delivery system format on bioaccessibility: nanocrystals, nanoemulsion droplets, and natural oil bodies, Food Funct, 10 (2019) 4339-4349.

[21] N.R. Goud, K. Suresh, P. Sanphui, A. Nangia, Fast dissolving eutectic compositions of curcumin, International journal of pharmaceutics, 439 (2012) 63-72.

[22] L. Shen, C.C. Liu, C.Y. An, H.F. Ji, How does curcumin work with poor bioavailability? Clues from experimental and theoretical studies, Scientific reports, 6 (2016).

[23] F. Paciello, A. Rita Fetoni, D. Mezzogori, R. Rolesi, A. Di Pino, G. Paludetti, C. Grassi, D. Troiani, The dual role of curcumin and ferulic acid in counteracting chemoresistance and cisplatin-induced ototoxicity, Scientific reports, 10 (2020) 1063.

[24] P. Benito-Gallo, A. Franceschetto, J.C.M. Wong, M. Marlow, V. Zann, P. Scholes, P. Gershkovich, Chain length affects pancreatic lipase activity and the extent and $\mathrm{pH}$-time profile of triglyceride lipolysis, European Journal of Pharmaceutics and Biopharmaceutics, 93 (2015) 353-362.

[25] M.T. Zeuner, T. Vallance, S. Vaiyapuri, G.S. Cottrell, D. Widera, Development and Characterisation of a Novel NF-kappaB Reporter Cell Line for Investigation of Neuroinflammation, Mediators of inflammation, 2017 (2017) 6209865.

[26] X. Feng, Q. Zhou, C. Liu, M.L. Tao, Drug screening study using glioma stem-like cells, Mol Med Rep, 6 (2012) 1117-1120. [27] A.T. Parsa, J.S. Waldron, A. Panner, C.A. Crane, I.F. Parney, J.J. Barry, K.E. Cachola, J.C. Murray, T. Tihan, M.C. Jensen, P.S. Mischel, D. Stokoe, R.O. Pieper, Loss of tumor suppressor PTEN function increases B7-H1 expression and immunoresistance in glioma, Nat Med, 13 (2007) 84-88.

[28] A. Zeng, J. Yin, Y. Li, R. Li, Z. Wang, X. Zhou, X. Jin, F. Shen, W. Yan, Y. You, miR-129-5p targets Wnt5a to block PKC/ERK/NFkappaB and JNK pathways in glioblastoma, Cell Death Dis, 9 (2018) 394.

[29] E. Liu, J. Wu, W. Cao, J. Zhang, W. Liu, X. Jiang, X. Zhang, Curcumin induces G2/M cell cycle arrest in a p53-dependent manner and upregulates ING4 expression in human glioma, J Neurooncol, 85 (2007) 263-270.

[30] X. Meng, J. Cai, J. Liu, B. Han, F. Gao, W. Gao, Y. Zhang, J. Zhang, Z. Zhao, C. Jiang, Curcumin increases efficiency of gamma-irradiation in gliomas by inhibiting Hedgehog signaling pathway, Cell Cycle, 16 (2017) 1181-1192.

[31] L. Wang, X. Ye, X. Cai, J. Su, R. Ma, X. Yin, X. Zhou, H. Li, Z. Wang, Curcumin suppresses cell growth and invasion and induces apoptosis by down-regulation of Skp2 pathway in glioma cells, Oncotarget, 6 (2015) 18027-18037.

[32] V. Soubannier, S. Stifani, NF-kappaB Signalling in Glioblastoma, Biomedicines, 5 (2017).

[33] X. Wang, L. Jia, X. Jin, Q. Liu, W. Cao, X. Gao, M. Yang, B. Sun, NF-kappaB inhibitor reverses temozolomide resistance in human glioma TR/U251 cells, Oncol Lett, 9 (2015) 2586-2590.

[34] S.K. Jha, S. Dey, R. Karki, Microemulsions- Potential Carrier for Improved Drug Delivery, Asian Journal of Biomedical and Pharmaceutical Sciences, 1 (2011) 5-9.

[35] P. Depraétere, A.T. Florence, F. Puisieux, M. Seiller, Some properties of oil-in-water emulsions stabilized with mixed nonionic surfactants (Brij 92 and Brij 96), International journal of pharmaceutics, 5 (1980) 291-304.

[36] H. Yamashita, Y. Hirakura, M. Yuda, T. Teramura, K. Terada, Detection of cocrystal formation based on binary phase diagrams using thermal analysis, Pharm Res, 30 (2013) 70-80.

[37] P. Wasutrasawat, H. Al-Obaidi, S. Gaisford, M.J. Lawrence, W. Warisnoicharoen, Drug solubilisation in lipid nanoparticles containing high melting point triglycerides, European journal of pharmaceutics and biopharmaceutics : official journal of Arbeitsgemeinschaft fur Pharmazeutische Verfahrenstechnik e.V, 85 (2013) 365-371.

[38] C. Malcolmson, M.J. Lawrence, A comparison of the incorporation of model steroids into non-ionic micellar and microemulsion systems, The Journal of pharmacy and pharmacology, 45 (1993) 141-143.

[39] S. Mondal, S. Ghosh, S. Moulik, Stability of curcumin in different solvent and solution media: UV-visible and steady-state fluorescence spectral study., Journal of Photochemistry and Photobiology B, 158 (2016) 212-218.

[40] L. Hu, Y. Jia, F. Niu, Z. Jia, X. Yang, K. Jiao, Preparation and enhancement of oral bioavailability of curcumin using 
[41] S. Calligaris, F. Valoppi, L. Barba, L. Pizzale, M. Anese, L. Conte, M.C. Nicoli, Development of Transparent Curcumin 768 Loaded Microemulsions by Phase Inversion Temperature (PIT) Method: Effect of Lipid Type and Physical State on Curcumin 769 Stability, Food Biophysics, 12 (2017) 45-51.

770 [42] Y.J. Wang, M.H. Pan, A.L. Cheng, L.I. Lin, Y.S. Ho, C.Y. Hsieh, J.K. Lin, Stability of curcumin in buffer solutions and 771 characterization of its degradation products, J Pharm Biomed Anal, 15 (1997) 1867-1876.

772 [43] C. Schneider, O.N. Gordon, R.L. Edwards, P.B. Luis, Degradation of Curcumin: From Mechanism to Biological Implications, 773 Journal of agricultural and food chemistry, 63 (2015) 7606-7614.

774 [44] J. Wang, X. Xu, H. Chen, S.S. Zhang, Y.X. Peng, Oxidation of Sodium Deoxycholate Catalyzed by Gold Nanoparticles and 775 Chiral Recognition Performances of Bile Salt Micelles, Molecules, 24 (2019).

776 [45] O.N. Gordon, C. Schneider, Vanillin and ferulic acid: not the major degradation products of curcumin, Trends in molecular 777 medicine, 18 (2012) 361-363; author reply 363-364.

778 [46] F. Payton, P. Sandusky, W.L. Alworth, NMR study of the solution structure of curcumin, Journal of natural products, 70 779 (2007) 143-146.

780 [47] P. Suresh, K. Gurudutt, K. Srinivasan, Degradation of bioactive spice compound: Curcumin during domestic cooking, 781 European Food Research and Technology, 228 (2009) 807-812.

782 [48] J. Lee, M.H. Rhee, E. Kim, J.Y. Cho, BAY 11-7082 is a broad-spectrum inhibitor with anti-inflammatory activity against 783 multiple targets, Mediators of inflammation, 2012 (2012) 416036.

784 [49] M. Esmaili, S.M. Ghaffari, Z. Moosavi-Movahedi, M.S. Atri, A. Sharifizadeh, M. Farhadi, R. Yousefi, J.-M. Chobert, T. 785 Haertlé, A.A. Moosavi-Movahedi, Beta casein-micelle as a nano vehicle for solubility enhancement of curcumin; food industry 786 application, LWT - Food Science and Technology, 44 (2011) 2166-2172.

787 [50] F. Zhang, G.Y. Koh, D.P. Jeansonne, J. Hollingsworth, P.S. Russo, G. Vicente, R.W. Stout, Z. Liu, A novel solubility-enhanced 788 curcumin formulation showing stability and maintenance of anticancer activity, Journal of pharmaceutical sciences, 100 789 (2011) 2778-2789.

790 [51] W. Liu, X.D. Chen, Z. Cheng, C. Selomulya, On enhancing the solubility of curcumin by microencapsulation in whey 791 protein isolate via spray drying, Journal of Food Engineering, 169 (2016) 189-195. 


\section{Captions to Figures}

Figure 1: Schematic showing the composition of formed microemulsions encapsulating CUR/FA eutectic mixtures and stabilised by Brijß 010/sodium deoxycholate or Brijß $\mathrm{O} 10$ only.

Figure 2: Pseudo-ternary phase diagrams of glyceryl tricaprate (a), (b), (c), (d) and glyceryl trioleate (e), (f), (g), (h) with Brijß $\mathrm{O} 10$ and sodium deoxycholate (SD) as the surfactants system. Colours indicate formed structures as microemulsions (red), nanoemulsions (yellow), emulsions (blue) and two phases/gels (grey). Data are means + SD of at least three experiments $(n=3)$.

Figure 3: Particle size analysis of microemulsions formed from solid and liquid oils using Brijß O10 (B) and oil (O); the symbols $\left({ }^{*}\right),\left({ }^{* *}\right),\left({ }^{* * *}\right),(+)$ indicate no statistical difference among annotated groups, the rest of data sets are statistically different $(p<0.05)$ and zeta potential measurements of microemulsions containing various ratio of oil (liquid) and surfactant Brijß 010; the symbol $\left(^{*}\right)$ indicates a statistical difference while $(x)$ indicates no statistical difference $(p<0.05)$. A total of 10 measurements were collected for each sample. Data are means \pm SD compared to measurements within the same and different data set, analysis was performed based on one-way analysis of variance (ANOVA), Tukey's post-hoc analysis using SPSS v25.

Figure 4: DSC thermogram showing the melting endotherm of curcumin and ferulic acid and their eutectic mixtures. The XRPD scans confirm DSC results showing lack of new emerging peaks. All measurements were repeated in triplicate $(n=3)$.

Figure 5: DSC thermograms of curcumin or curcumin/ferulic acid in glyceryl tricaprate microemulsion (a) and glyceryl trioleate microemulsion (b). All measurements were repeated in triplicate $(n=3)$. 
Figure 6: DSC thermograms of CUR or CUR/FA dissolved in glyceryl tricaprate (solid oil) showing pure components and eutectic mixture. All measurements were repeated in triplicate $(n=3)$.

Figure 7: Particle size analysis of microemulsions with CUR or CUR/FA eutectic mixture $\left({ }^{*} p<0.05\right.$ : microemulsions with eutectic mixtures are significantly different to microemulsions alone) $\left({ }^{* *} p<0.05\right.$ : Microemulsions with CUR are significantly different to microemulsions alone $) \quad\left({ }^{* *} p<0.05\right.$ : Microemulsions with CUR are significantly different to microemulsions alone and microemulsions with eutectic mixtures) $\left({ }^{* * *} p<0.05\right.$ : Microemulsions with eutectic mixtures are significantly different to microemulsions alone and microemulsions with CUR). A total of 10 measurements were collected for each sample. The size and morphology were further verified using cryo-SEM of CUR/FA microemulsion showing oil particles wrapped within the surfactant network.

Figure 8: solubility measurements of CUR in microemulsions prepared using Brij® $\mathrm{O} 10$ as the only surfactant. Microemulsions were prepared using the glyceryl tricaprate (solid oil) and glyceryl trioleate (liquid oil). All measurements were repeated in triplicate $(n=3)$. The symbols $\left({ }^{*}\right)$ or $(+)$ indicate no statistical difference $(p<0.05)$, all other data sets are statistical different $(p<0.05)$. Data are means $\pm S D$ compared to measurements within same and different data set, analysis was performed based on one-way analysis of variance (ANOVA), Tukey's post-hoc analysis using SPSS v25.

Figure 9: Solubility of curcumin in microemulsions prepared using (a) solid oil glyceryl tricaprate and (b) liquid oil glyceryl trioleate. Measurements were performed immediately and after 21 days of preparation. No statistical difference between immediate measurements and measurements made after 21 days of preparation $(p<0.05 \%)$. All measurements were repeated in triplicate $(n=3)$. Data are means \pm SD compared to measurements within same and different data set, analysis was performed based on one-way analysis of variance (ANOVA), Tukey's post-hoc analysis using SPSS v25. 
Figure 10: Degradation of CUR measured using UV photospectroscopy after dilution in DMSO and phosphate buffer ( $\mathrm{pH} 7.5)$, degassed phosphate buffer $(\mathrm{pH} 7.5)$, phosphate buffer $(\mathrm{pH} 7.5)$ and CUR/FA 1:1 physical mixture in phosphate buffer $(\mathrm{pH}$ 7.5). Measurements were collected at wavelength of $429 \mathrm{~nm}$ and at $37^{\circ} \mathrm{C}$. Concentration of CUR was $40 \mu \mathrm{M}$ in all measurements. All measurements were repeated in triplicate $(n=3)$.

Figure 11: Degradation of CUR measured at wavelength of $429 \mathrm{~nm}$ using photospectroscopy after exposure to methanol and phosphate buffer $(\mathrm{pH} \mathrm{7.5)} \mathrm{in}$ microemulsions prepared with $5 \%$ oil and $20 \%$ surfactant compared to CUR alone using (a) Liquid oil glyceryl trioleate. (b) Solid oil glyceryl tricaprate. ( ${ }^{*} p<0.05$ : All microemulsions with the addition of either CUR or CUR/FA are significantly different to CUR alone without incorporation into the microemulsions). (c $-f)$, Extracted ion chromatograms for CUR (top) and FA (bottom) from 7\% Brijß 010, 1\% solid glyceryl tricaprate (c), 7\% Brijß 010, 1\% liquid glyceryl trioleate (d), 7\% Brijß 010, 1\% solid glyceryl tricaprate CUR:FA (1:1) (e) and 7\% Brij® 010, 1\% liquid glyceryl trioleate CUR:FA (1:1) (f). All measurements were performed at ambient temperature. All measurements were repeated at least in triplicate $(n=3)$. Data are means $\pm S D$ compared to measurements within same and different data set.

Figure 12: Characteristic region of ${ }^{1} \mathrm{H}$ NMR spectrum of CUR and FA alone or loaded within the formed microemulsions. Comparison between spectra of intact CUR and FA (red box indicates characteristic resonances) is shown in (a), whereas the comparison between spectra of CUR and microemulsion is shown in (b). CUR spectra recorded at elevated temperatures are shown in (c).

Figure 13: U251-NF-KB-GFP-Luc cells were exposed to TNF- $\alpha$ or LPS followed by a subsequent assessment of NF-kB-dependent luciferase activity. A) U251-NF-kBGFP-Luc co-exposed to TNF- $\alpha$ and microemulsion of CUR/FA show significantly lower levels of NF-kB activity when compared to cells exposed either to TNF- $\alpha$ alone or to a combination of TNF- $\alpha$ and CUR. B) Microemulsion of CUR/FA decrease the LPSinduced activity of NF-KB compared to LPS alone or a combination of CUR and LPS. Data is presented as mean \pm standard deviation from at least three independent 
experiments. ${ }^{*} p<0.05,{ }^{* *} p<0.01$, and ${ }^{* * *} p<0.001$ were considered significant (ANOVA with Bonferroni correction, Cl 95\%). C) CUR/FA microemulsions after storage for 12 months at ambient conditions. All samples contained similar concentration of CUR and had similar color intensity when were freshly prepared.

Supplementary Figure 1: U251 cells cultivated in the presence of increasing concentrations of CUR for 48 hours followed by XTT-based assessment of cell viability. Cells showed no significant difference in viability below $400 \mu \mathrm{M}$ of CUR. B). U251 cells cultivated in the presence of increasing concentrations of Curcumin 1 (7\% C18:1E10, 1\% solid glyceryl tricaprate). Cells showed no significant drop in viability below $50 \mu \mathrm{M}$ of Curcumin 1. All experiments were performed in triplicate. $\left({ }^{\star} P<0.05\right.$ ) 


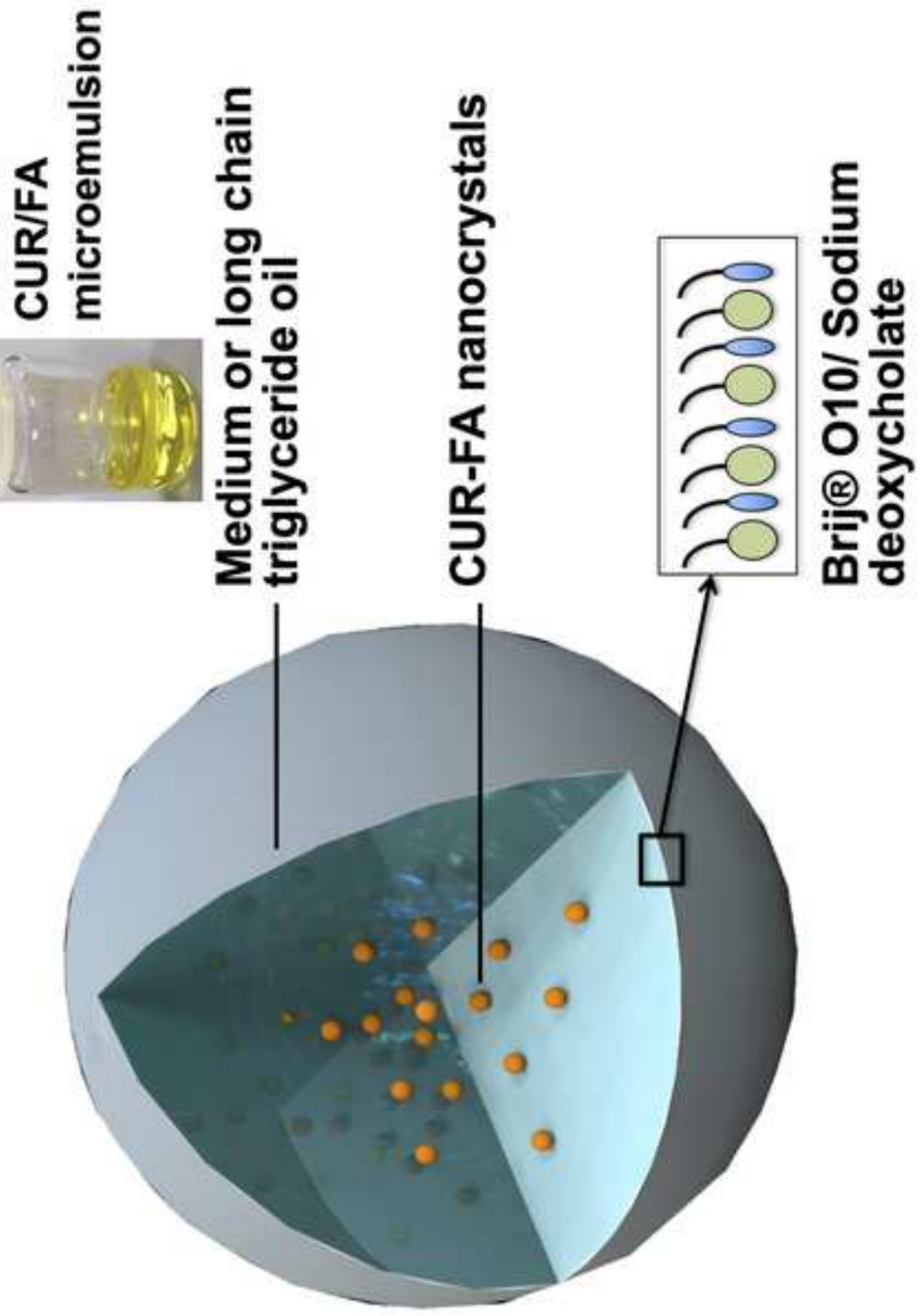

产 


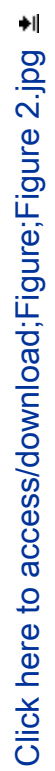

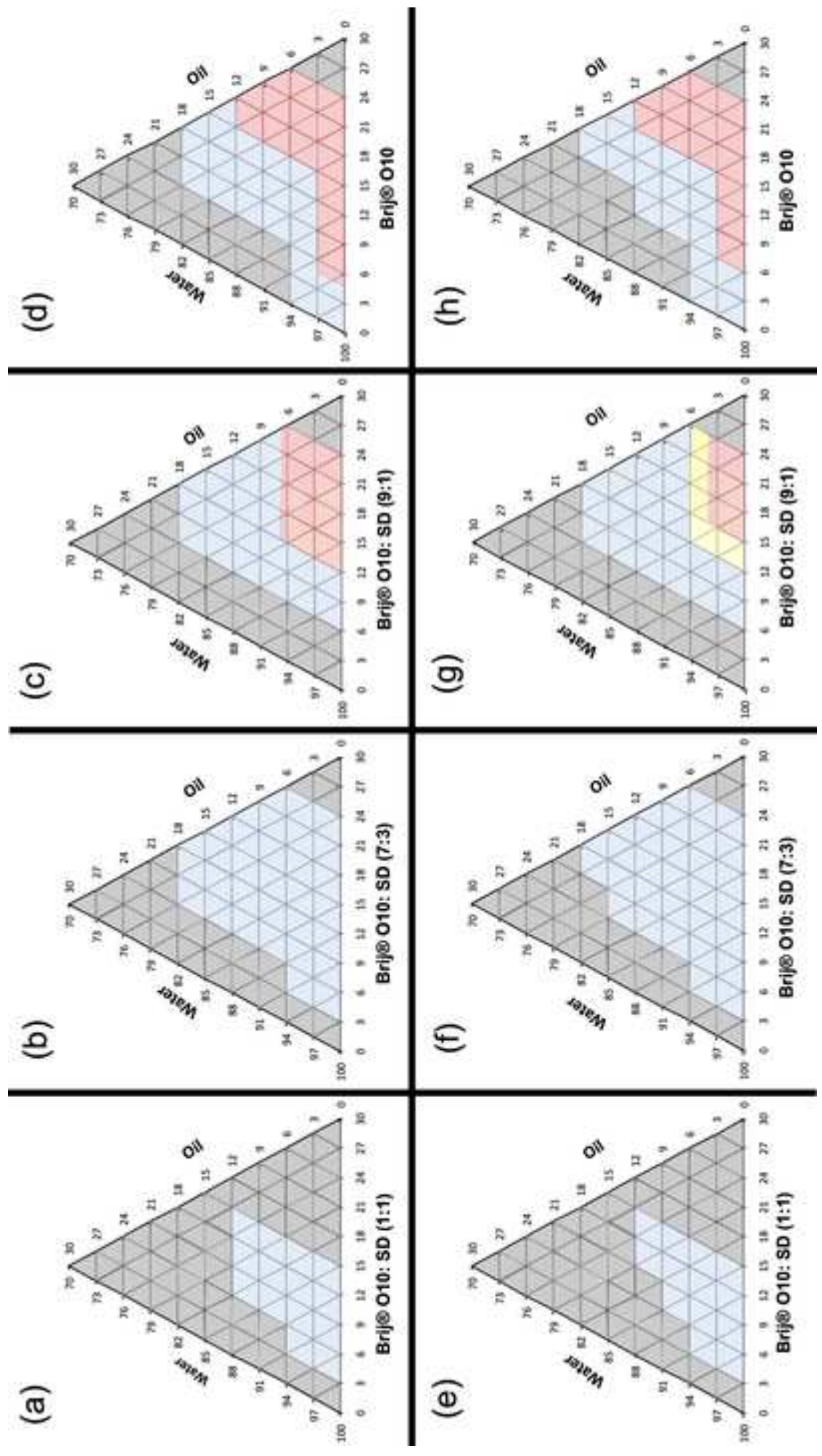

№ 

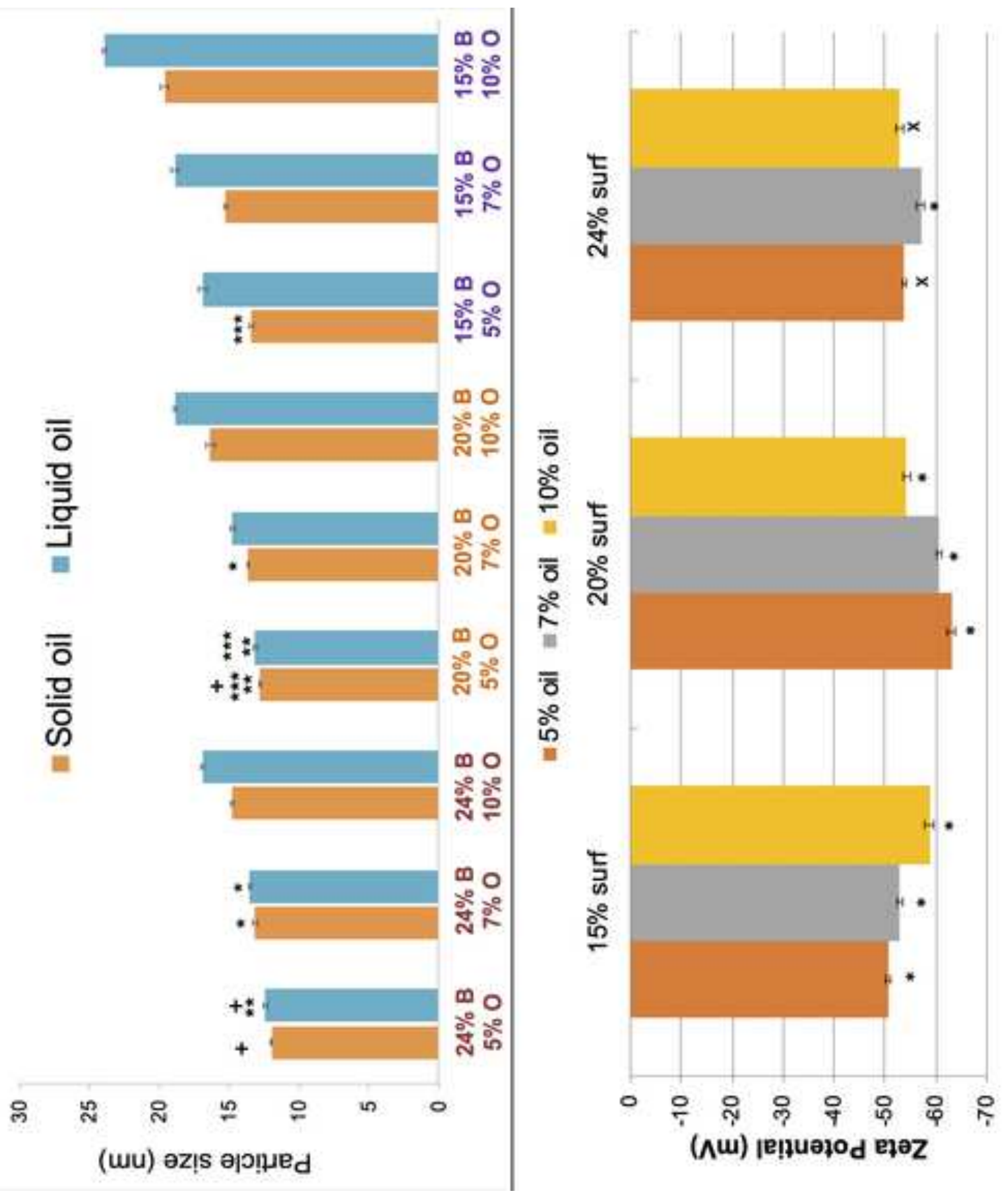

m
는
믄 


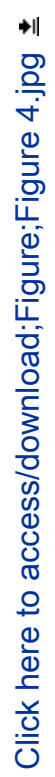

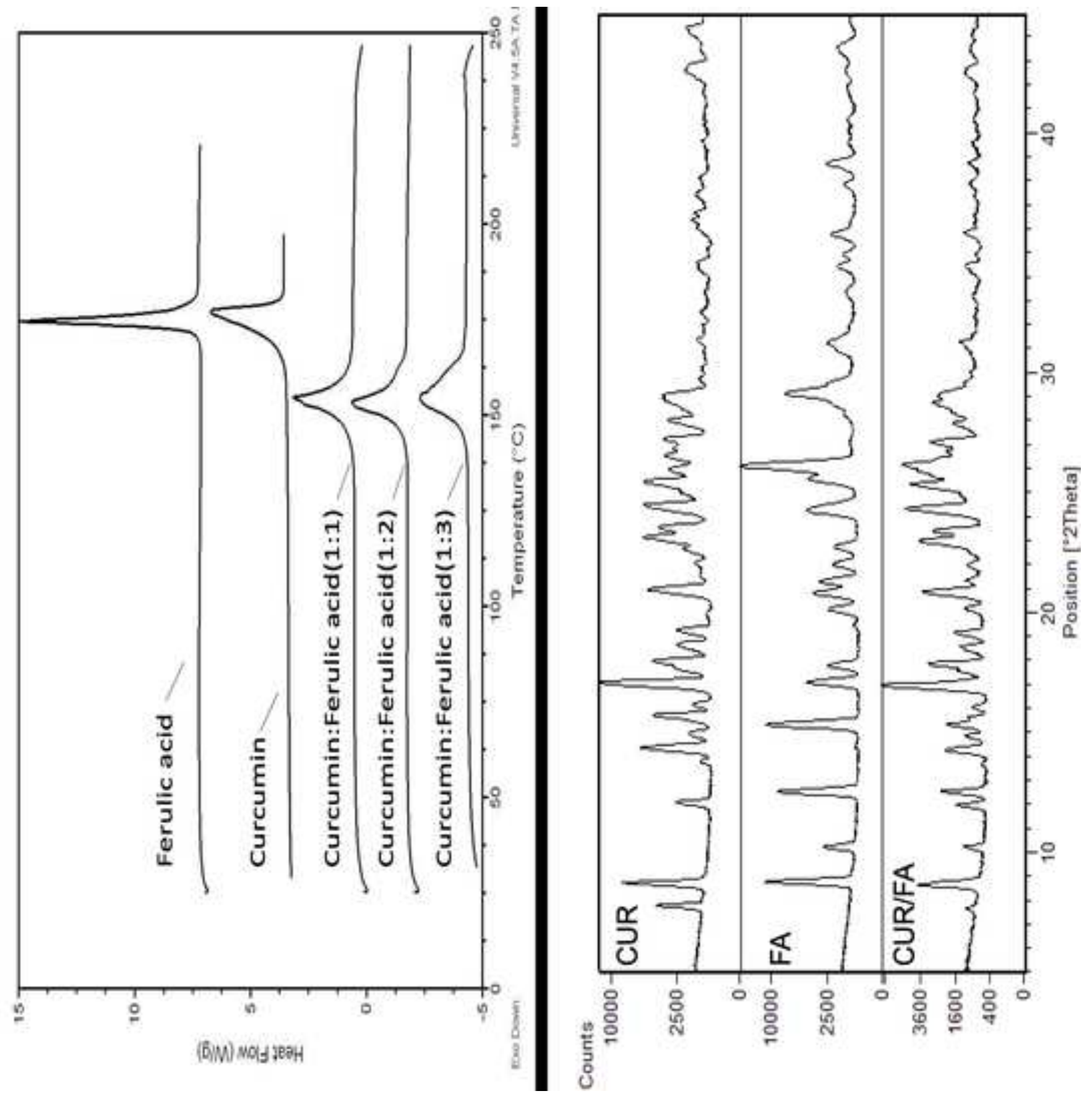

$\stackrel{+}{Ð}$ 


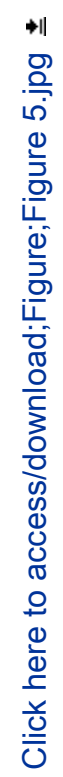

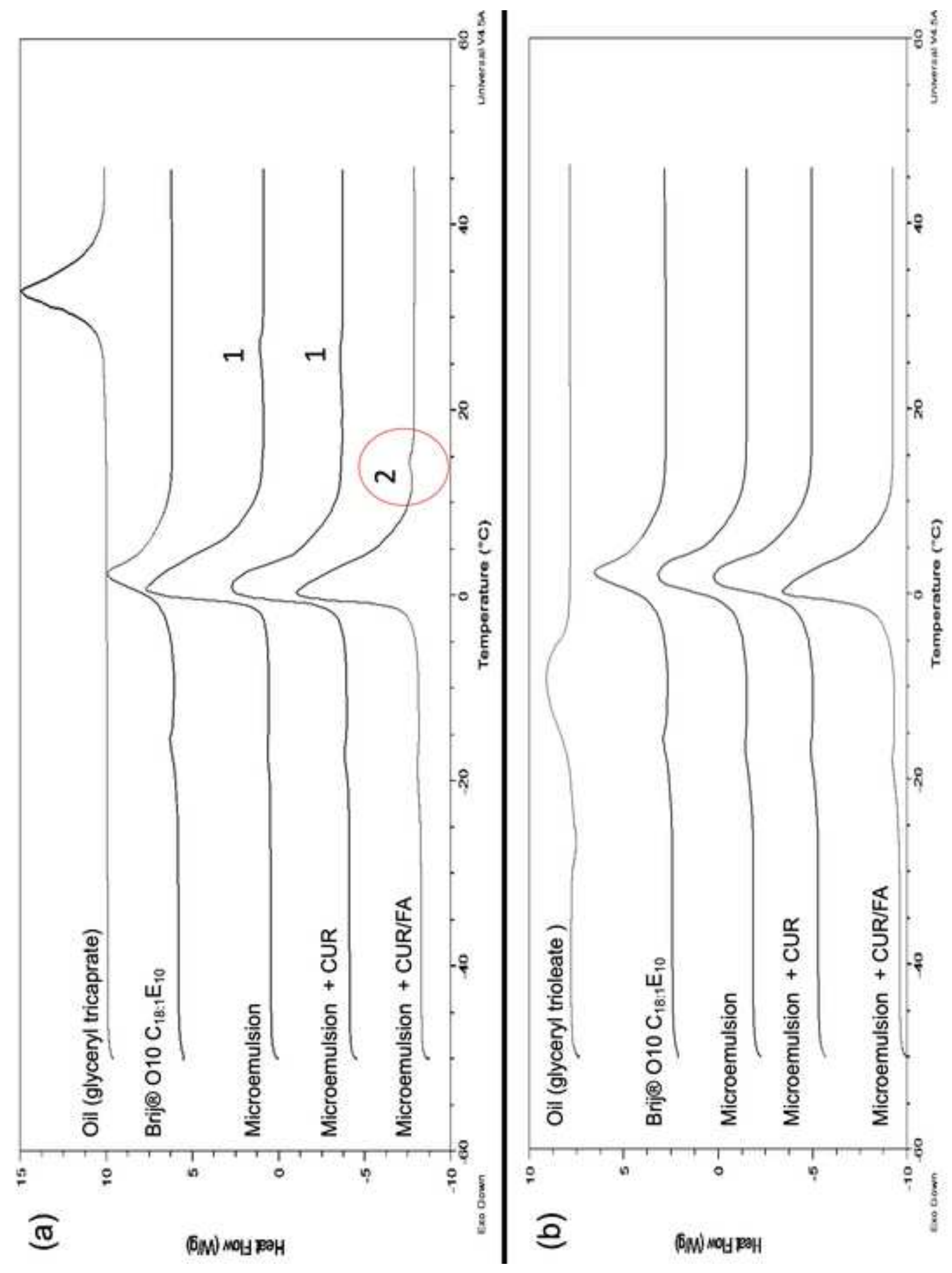

竞 


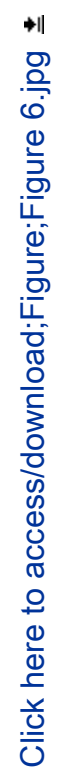

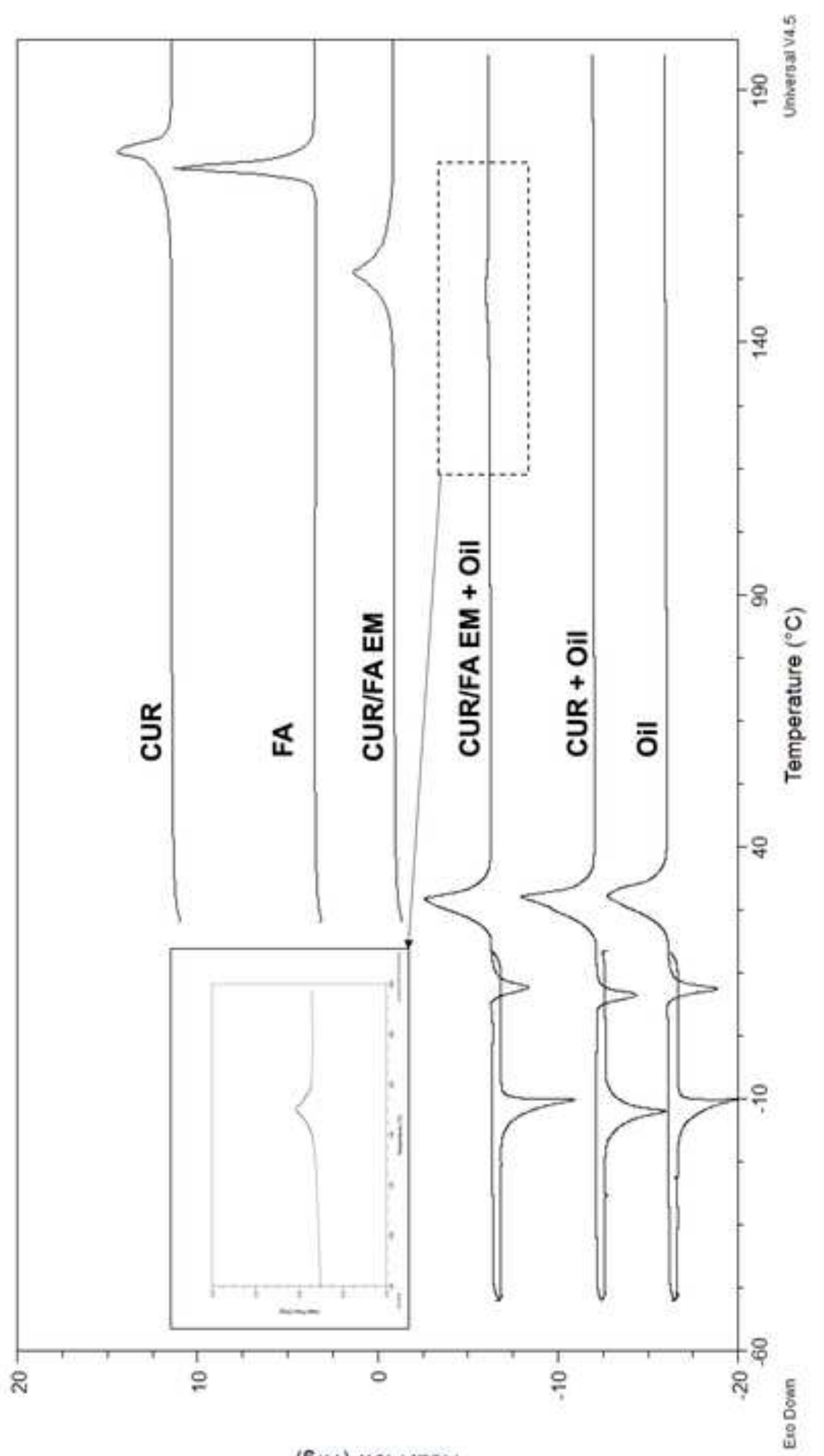

0
0
$\stackrel{0}{3}$
흔

(6/M) M이 IEaH 


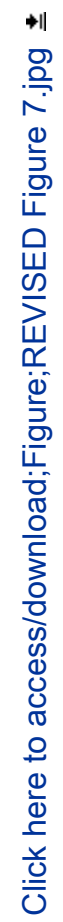
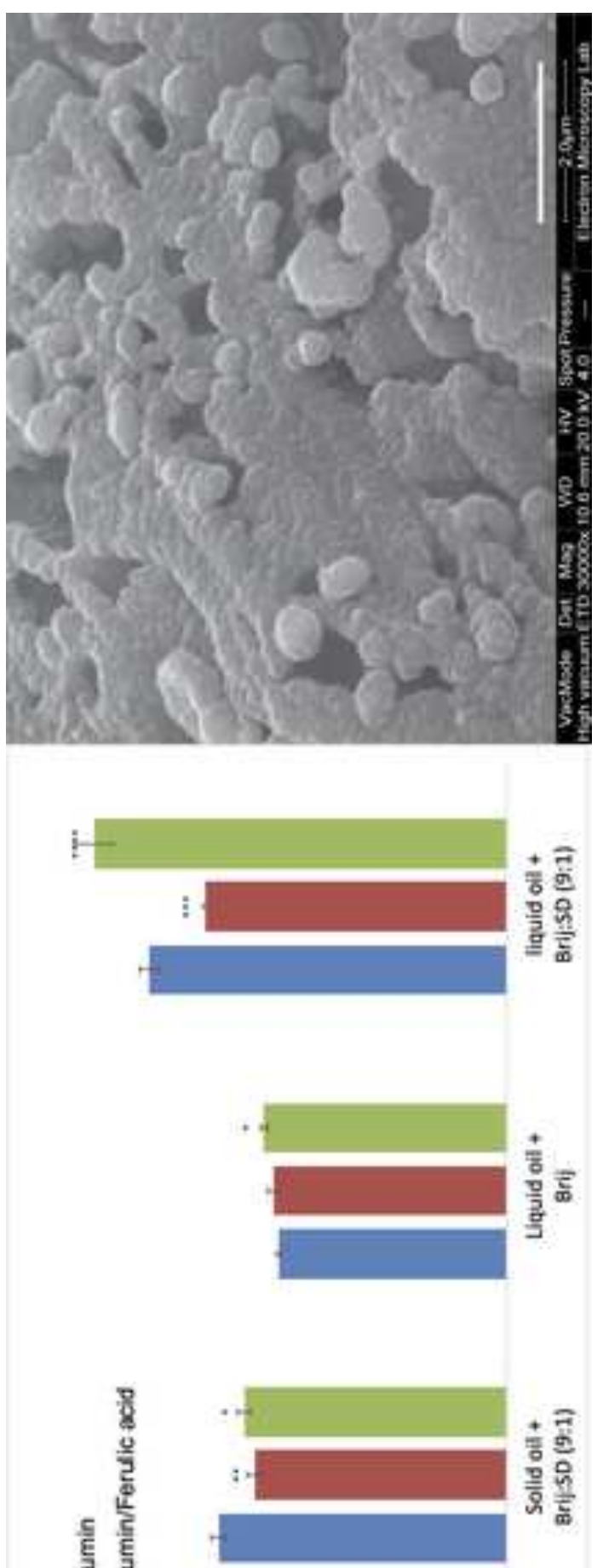

군 


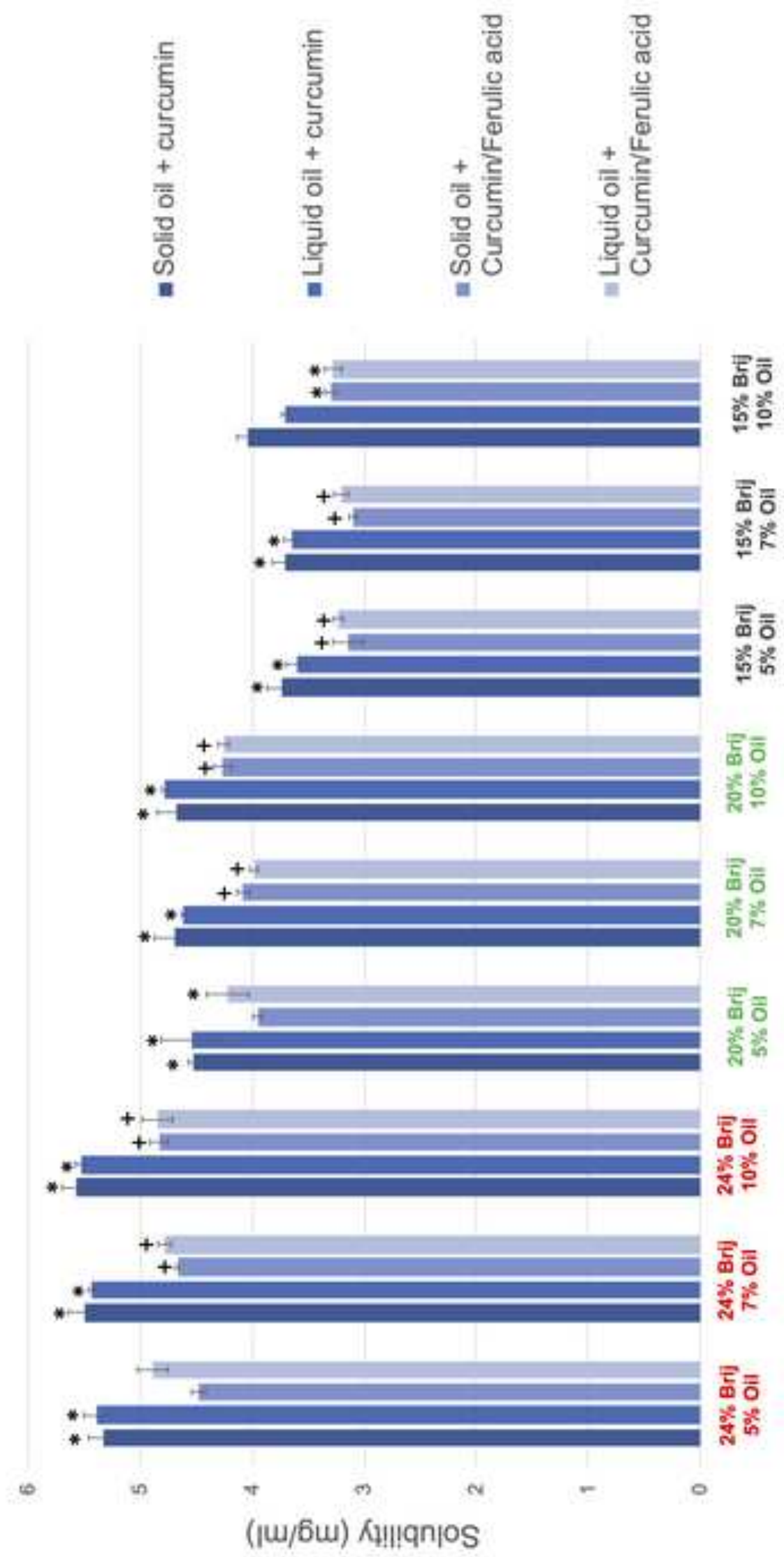

$\infty$
는
믄 


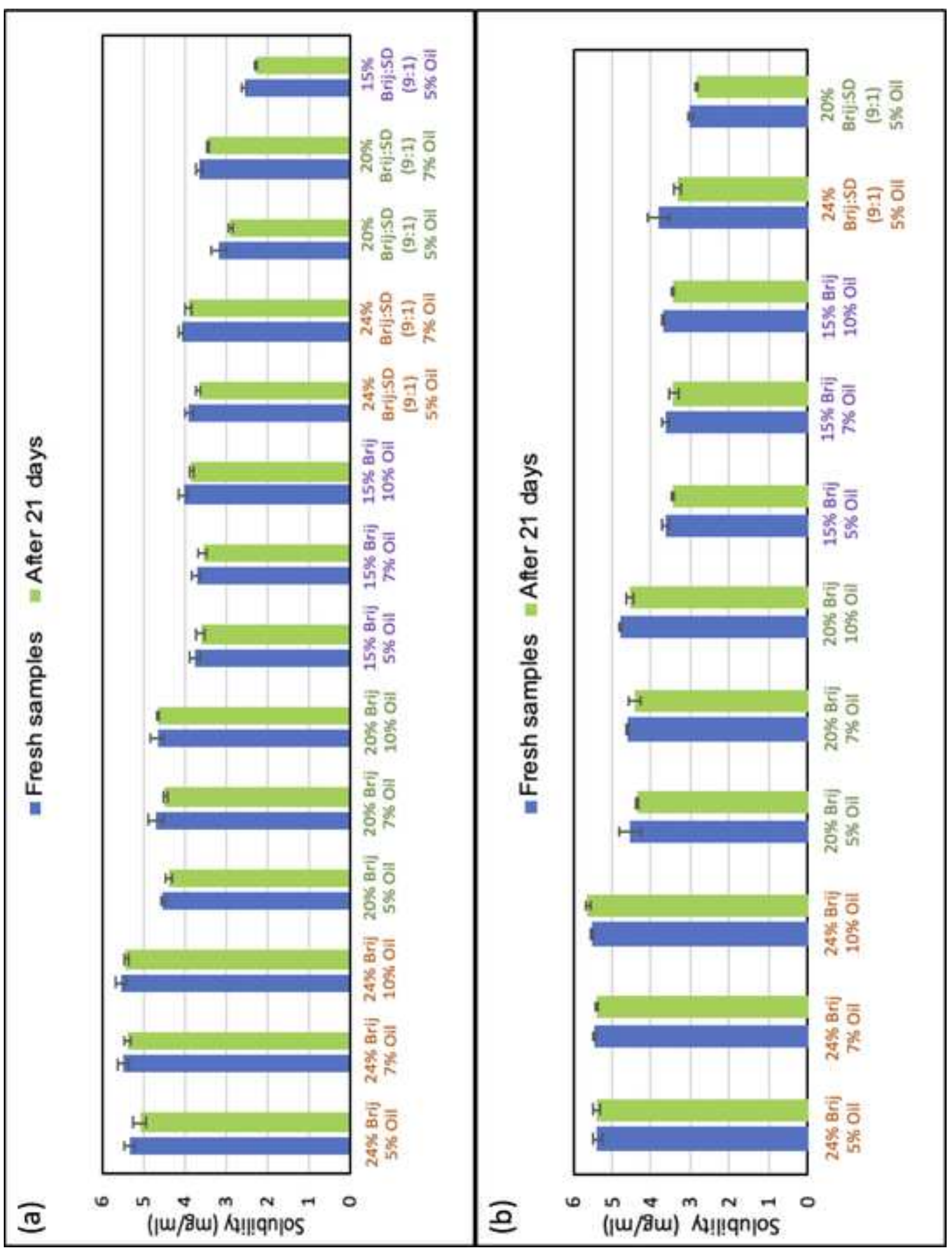

몬 


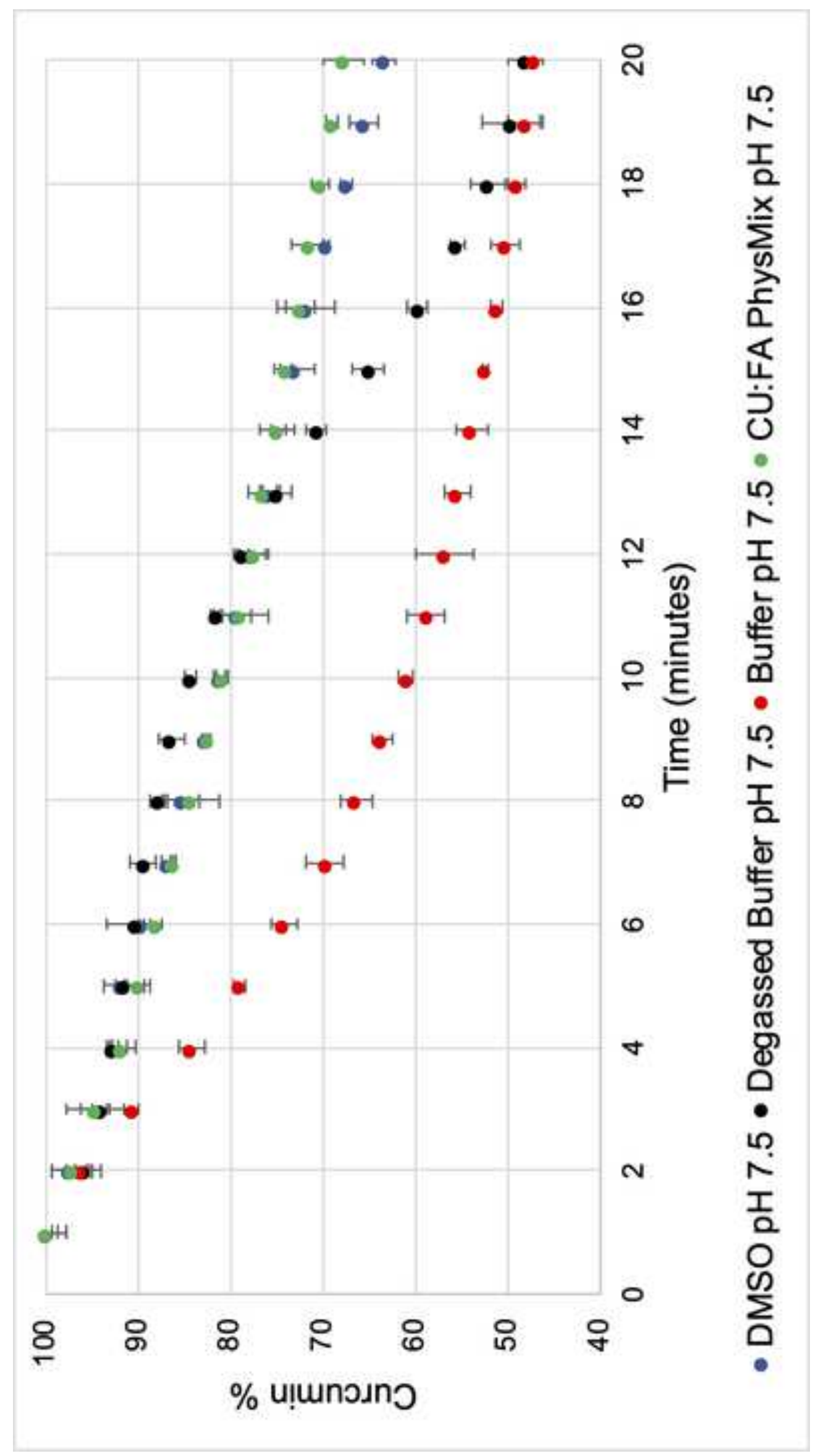

음 


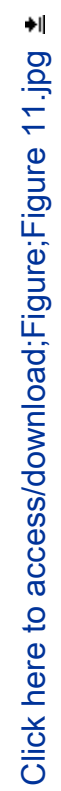
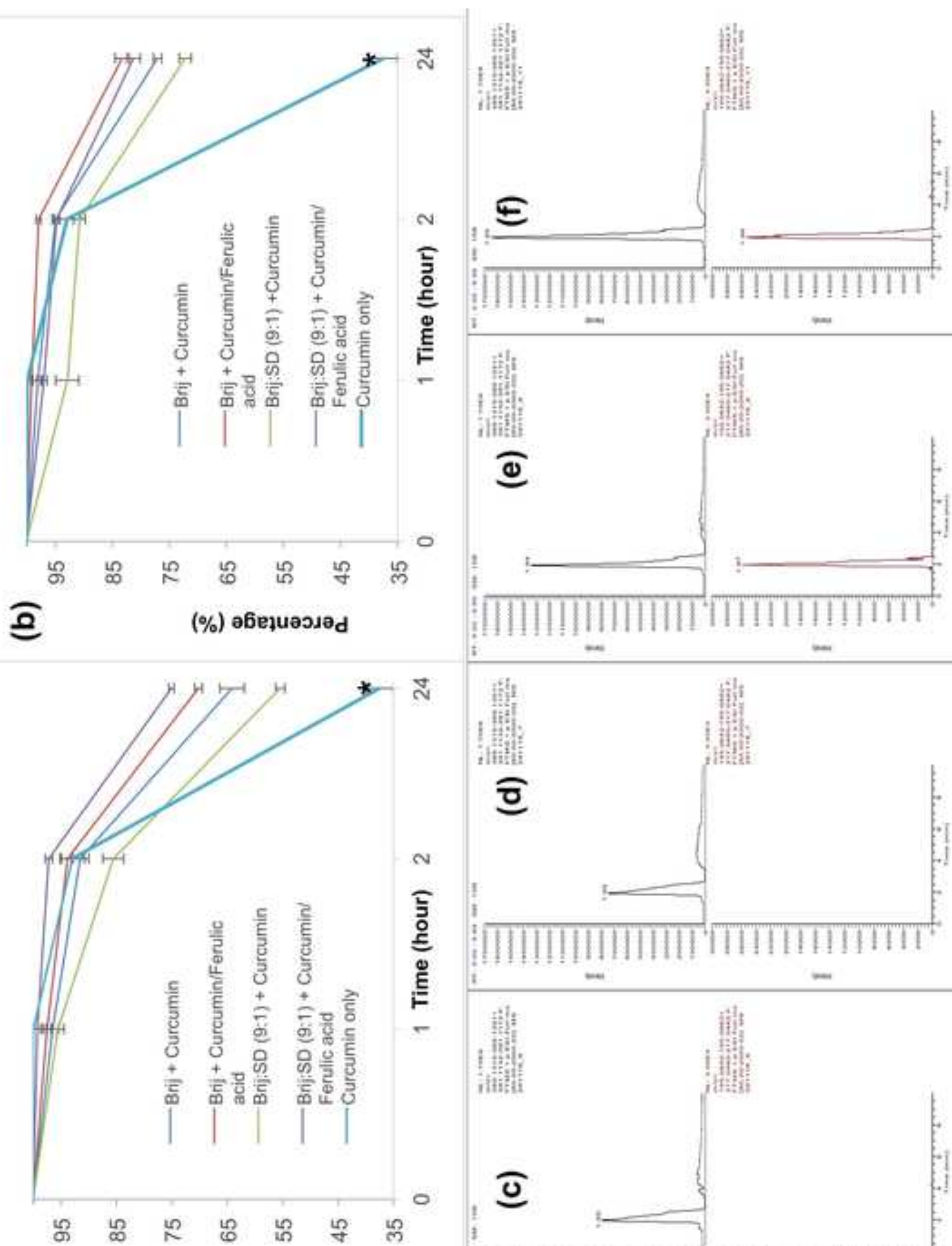

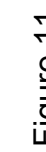

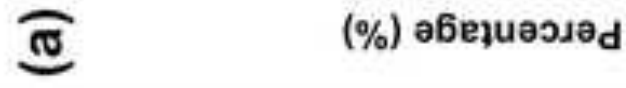




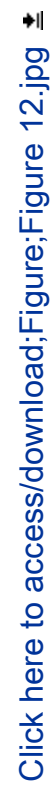

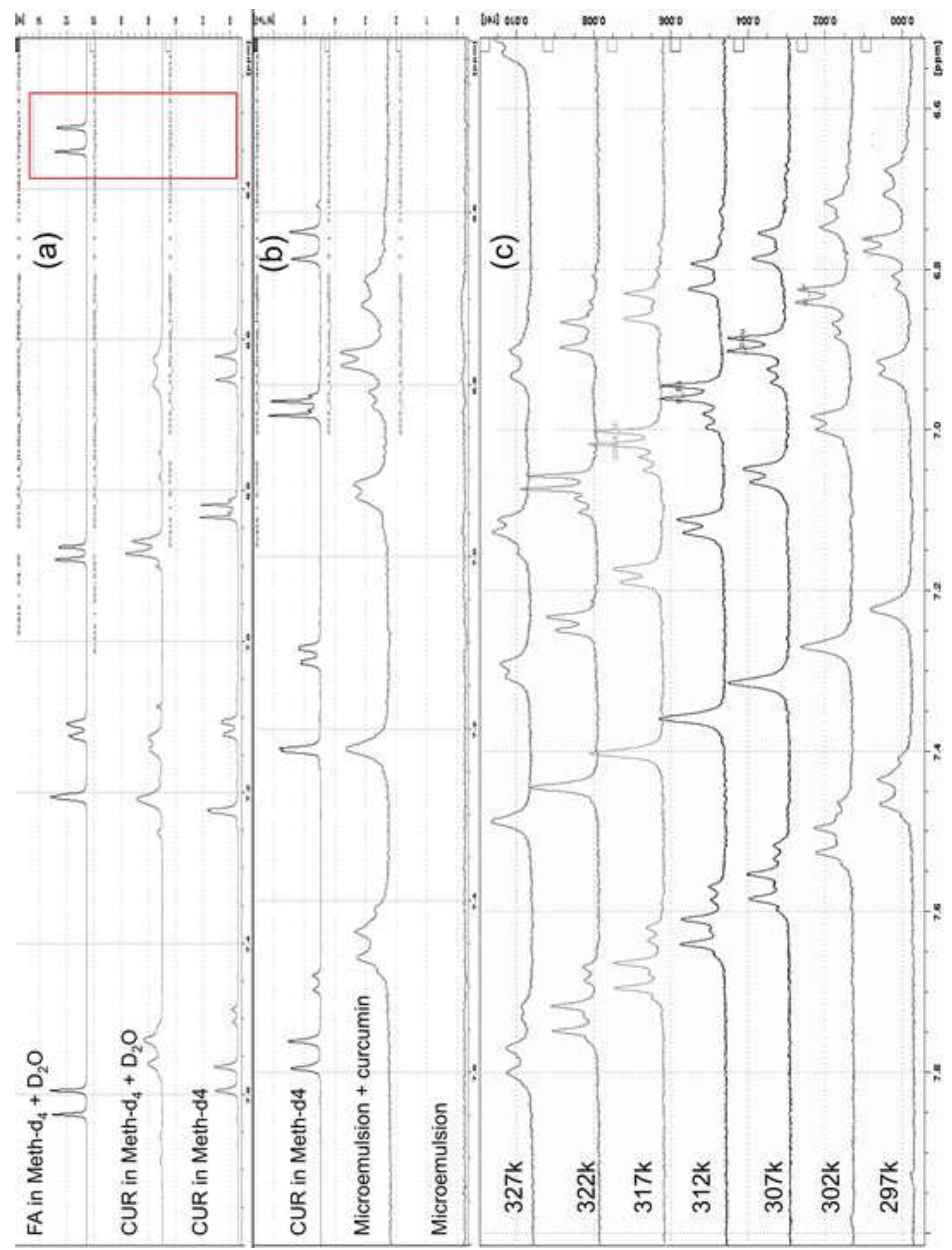

훈 

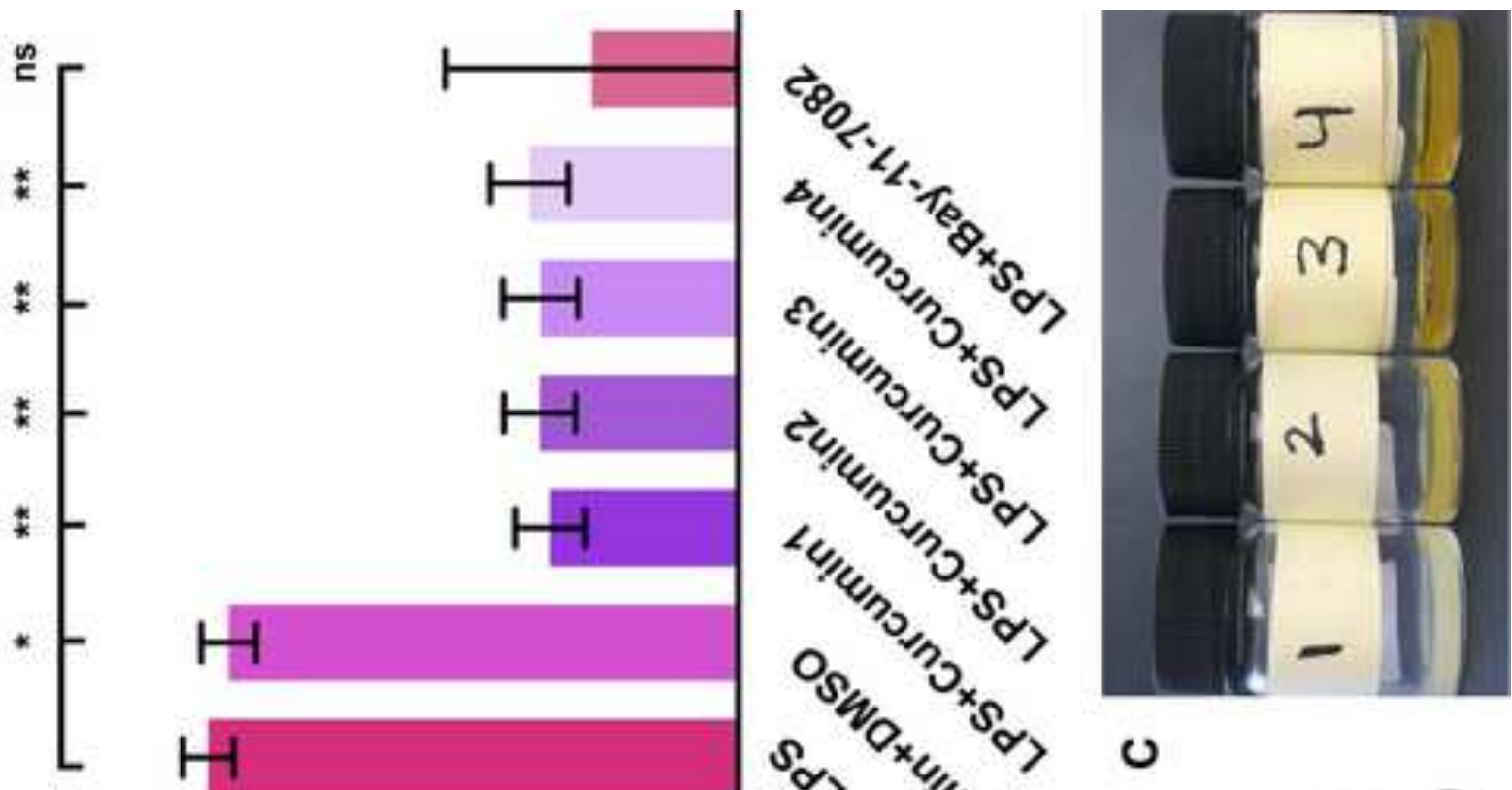

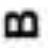
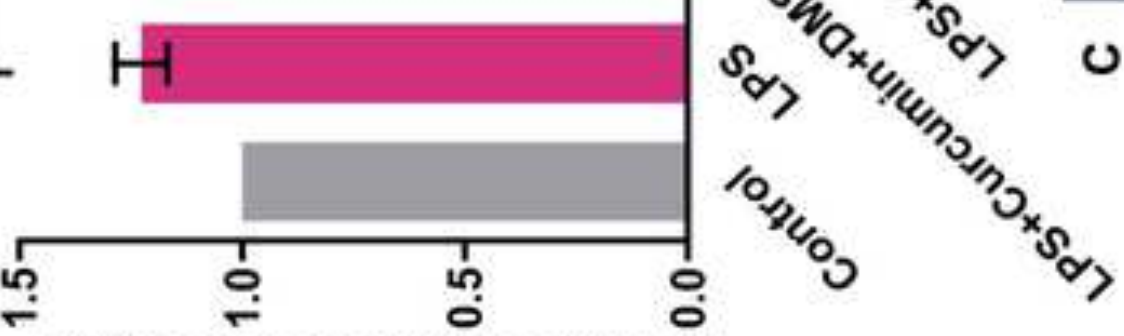

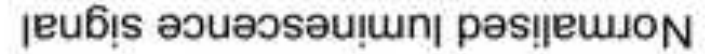

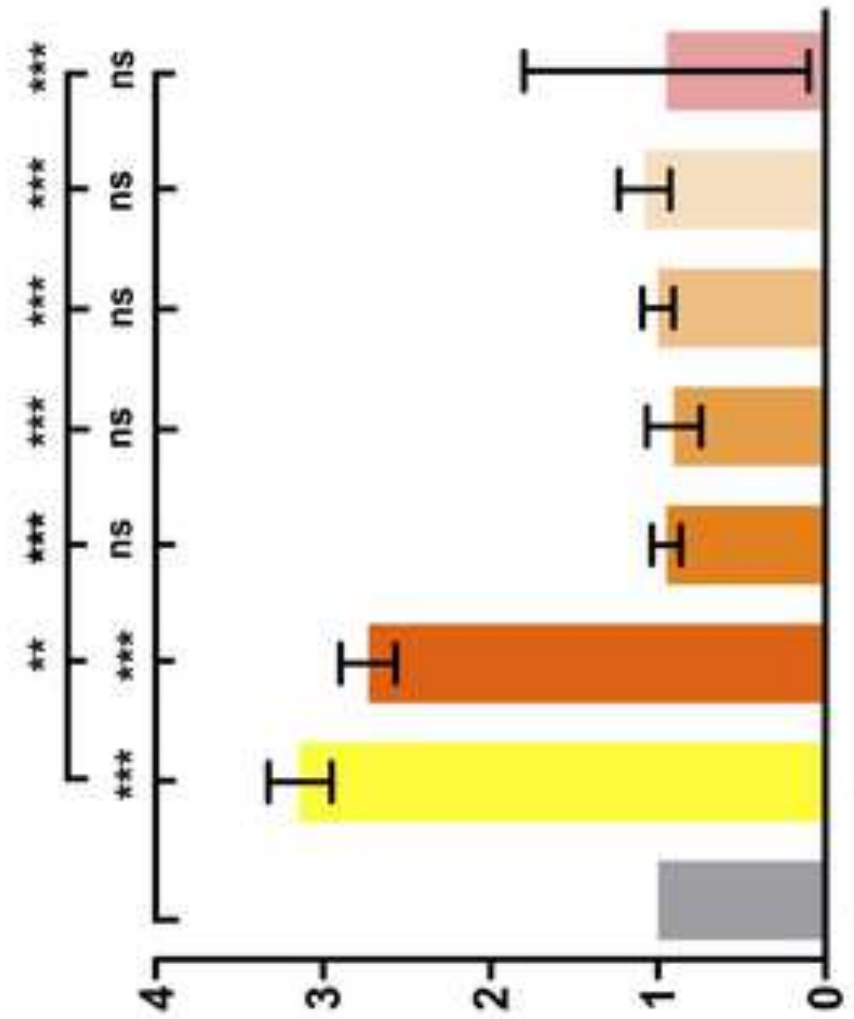

$\varangle$

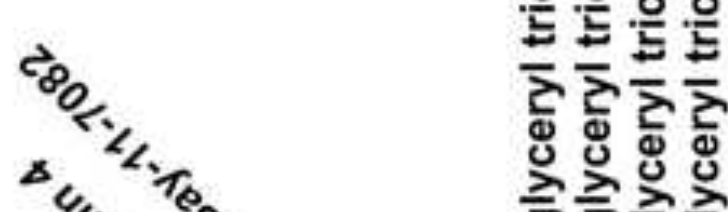

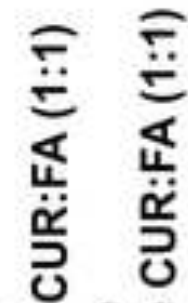
๑ 뜽응 응 은 은 은 은

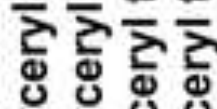

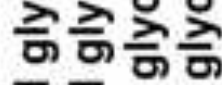
믁 믁 므으 프믐 응 ㅇํㅇํํㅇำ $r+r$ 웅유움 แய山ய $5 \div \div$ $\ddot{\infty} \ddot{\infty} \ddot{\infty}$ 5555 웅ㅇㅇ응 イイr $\ddot{\sim} \dot{v} \ddot{\dot{q}}$ 들드ㄹㅡㅡㄴ 든 


\section{Author Statement}

Hisham Al-Obaidi: Conceptualization, Project administration, Supervision, Writing original draft. Farah Sanduk: Investigation. Yiming Meng: Investigation. Darius Widera: Investigation. Radoslaw M.Kowalczyk: Investigation. Nicholas Michael: Investigation. Amanpreet Kaur:Investigation. Vivian Yip: Investigation. Sandra Zulu: Investigation. Irene Zavrou: Investigation. Lulu Hana: Investigation. Muhammad Yaqoob: Investigation. 
Click here to access/download Supplementary Material Supplementary Figure.jpg 
Click here to access/download Supplementary Material Appendix Table.docx 